\title{
HOMOLOGICAL DIMENSIONS AND ABELIAN MODEL STRUCTURES ON CHAIN COMPLEXES
}

\author{
MARCO A. PÉREZ
}

\begin{abstract}
We construct Abelian model structures on the category of chain complexes over a ring $R$, from the notion of homological dimensions of modules. Given an integer, $n>0$, we prove that the left modules over a ringoid $\mathfrak{R}$ with projective dimension at most $n$ form the left half of a complete cotorsion pair. Using this result, we prove that there is a unique Abelian model structure on the category of chain complexes over $R$, where the exact complexes are the trivial objects and the complexes with projective dimension at most $n$ form the class of trivially cofibrant objects. In [4], the authors construct an Abelian model structure on chain complexes, where the trivial objects are the exact complexes, and the class of cofibrant objects is given by the complexes whose terms are all projective. We extend this result by finding a new Abelian model structure with the same trivial objects where the cofibrant objects are given by the class of complexes whose terms are modules with projective dimension at most $n$. We also prove similar results concerning flat dimension.
\end{abstract}

1. Introduction. In 2002, Hovey established in [14, Theorem 2.2] a correspondence for constructing an Abelian model structure from two compatible and complete cotorsion pairs (see, [14, Theorem 2.2]). We apply this correspondence in order to construct new model structures on the category of chain complexes, from certain special classes of chain complexes involving homological dimensions.

In his book, [15, Section 2.3], Hovey constructs a model category structure on the category $\mathbf{C h}\left({ }_{R} \mathbf{M o d}\right)$ of chain complexes of left $R$ modules, where the weak equivalences are the quasi-isomorphisms and the trivial cofibrations are the monomorphisms whose cokernels are pro-

2010 AMS Mathematics subject classification. Primary 18G20, 18G35.

Keywords and phrases. Homological dimensions, chain complexes, Abelian model structures, cotorsion pairs, Hovey's correspondence.

This work has been financially supported by scholarships from ISM-CIRGET and Fondation de l'UQÀM.

Received by the editors on April 4, 2014, and in revised form on June 26, 2014. DOI:10.1216/RMJ-2016-46-3-951 
jective. We shall refer to this structure as the projective model structure. We extend this model structure to any projective dimension. Specifically, we shall find a model structure with the same weak equivalences where the trivial cofibrations are the monomorphisms whose cokernels are complexes with projective dimension $\leq n$, with $n$ a positive integer. At the beginning of Section 3 we give a description of these complexes as those exact complexes whose cycles have projective dimension $\leq n$.

The method we use for constructing the model structure mentioned above involves the study in Section 3 of the category of left modules over a ringoid $\mathfrak{R}$, denoted $\operatorname{Mod}(\mathfrak{R})$. If we consider the class $\mathcal{P}_{n}(\operatorname{Mod}(\mathfrak{R}))$ of $n$-projective modules over $\mathfrak{R}$, i.e., modules with projective dimension $\leq n$, then we show that every module in $\mathcal{P}_{n}(\operatorname{Mod}(\mathfrak{R}))$ is filtered by the class $\left(\mathcal{P}_{n}(\operatorname{Mod}(\mathfrak{R}))\right)^{\leq \kappa}$ of $n$-projective modules with cardinality $\leq \kappa$, where $\kappa$ is a fixed infinite regular cardinal satisfying a certain condition with respect to $\mathfrak{R}$. As a consequence, one has that $\mathcal{P}_{n}(\operatorname{Mod}(\mathfrak{R}))$ is the left half of a complete cotorsion pair. These facts in $\operatorname{Mod}(\mathfrak{R})$ are generalizations of [2, Proposition 4.1, Theorem 4.2]. Since the category $\mathbf{C h}\left({ }_{R}\right.$ Mod) of chain complexes of left $R$-modules is a particular example of $\operatorname{Mod}(\mathfrak{R})$, we have that the class of $n$-projective complexes is the left half of a complete cotorsion pair, which turns out to be compatible with another complete cotorsion pair whose left half is given by the class of differential graded $n$-projective complexes. In order to prove this last assertion we present in Proposition 4.3 a rather simple but interesting method that relates the completeness of two given compatible cotorsion pairs. Roughly speaking, we shall see that, in order to show the completeness of two compatible cotorsion pairs, it suffices to prove that either is complete.

As a consequence of Hovey's correspondence, we shall get the Abelian model structure described in Theorem 4.5 (the $n$-projective model structure). One interesting application of this model structure is given at the end of Section 4 and consists of finding another way to compute extension groups $\operatorname{Ext}_{R}^{i}(M, N)$ for every pair of left $R$-modules, $M$ and $N$. Recall that one normally computes $\operatorname{Ext}_{R}^{i}(M, N)$ by using either a left resolution of $M$ by projective modules or a right resolution of $N$ by injective modules. Somewhat surprisingly, there are many other ways to do it. This was pioneered by Gillespie in [9, Section 5] who proved that, in order to compute $\operatorname{Ext}_{R}^{i}(M, N)$, one can use a left resolution of $M$ by flat modules and simultaneously a right resolution of $N$ 
by cotorsion modules. We prove that one can also use a left resolution of $M$ by modules of projective dimension at most $n$. The disadvantage of doing so is that we use right resolutions of $N$ by a class of modules which is hard to describe. The same results apply to the $n$-flat and $n$ injective model structures (see Theorems 4.9 and 6.1), although in the $n$-injective one we use right resolutions of $N$ by modules of injective dimension $\leq n$.

In Section 5, we construct another model structure on $\mathbf{C h}\left({ }_{R} \mathbf{M o d}\right)$ from the class of $n$-projective modules. In [4], Bravo, et al., construct an Abelian model structure on $\mathbf{C h}\left({ }_{R} \mathbf{M o d}\right)$, where the trivial objects are the exact complexes and the class of cofibrant objects is given by the complexes with projective terms. Among the arguments given by the authors, there is a famous theorem by Kaplansky on projective modules, which states that every projective module is a direct sum of countably generated projective modules. We present an extension of Kaplansky's theorem to any projective dimension, in order to prove that every degreewise $(\mathrm{dw}) n$-projective complex (we mean a complex whose terms have projective dimension $\leq n$ ) is filtered by the class of chain complexes $X$ such that each module $X_{k}$ has a projective resolution of length $n$, where each projective term is written as a direct sum of countably generated projective modules over a countable set. We introduce the notion of nice sub-resolutions of $n$-projective modules to show that every exact $\mathrm{dw}-n$-projective complex is filtered by the class of exact dw- $n$-projective complexes with cardinality $\leq \kappa$, with $\kappa>\operatorname{Card}(R)$. This gives rise to an extension of some of the techniques developed in Bravo, et al. [4], to the class of $n$-projective modules, from which we obtain a new Abelian model structure on $\mathbf{C h}\left({ }_{R} \mathbf{M o d}\right)$ where the quasi-isomorphisms are weak equivalences and the monomorphisms with (exact) dw- $n$-projective cokernels are (trivial) cofibrations.

Section 6 is devoted to obtaining two new model category structures on $\mathbf{C h}\left({ }_{R} \mathbf{M o d}\right)$ from the class of modules with flat dimension $\leq n$ (see Theorems 6.1 and 6.2). This is motivated by early studies in the case $n=0$ developed by Aldrich, et al., in [1, Propositions 3.1, 4.1], where they proved that every flat complex is filtered by the class of flat complexes with cardinality $\leq \kappa$. They also proved a similar result for the class of complexes which have all their terms flat. We show how their techniques can be adapted to the case $n>0$ to prove analogous results for the class of $n$-flat complexes, i.e., complexes with 
flat dimension $\leq n$, and degreewise $n$-flat complexes, i.e., complexes which have all their terms with flat dimension $\leq n$. Later, we deduce that every chain complex can be covered by a complex with flat dimension $\leq n$. This latter result represents an extension of Enochs' Flat cover conjecture (which states that every module has a flat cover) to the category of chain complexes and for any flat dimension.

The new model structures obtained in this paper are not monoidal in general. However, at the end, we present an interesting application of a Künneth theorem to show that the degreewise projective and degreewise flat model structures on $\mathbf{C h}\left({ }_{R} \mathbf{M o d}\right)$ are monoidal in the case where $R$ is a commutative ring with weak dimension at most 1 .

2. Preliminaries. Given an Abelian category $\mathcal{C}$, two classes $\mathcal{A}$ and $\mathcal{B}$ of objects in $\mathcal{C}$ form a cotorsion pair $(\mathcal{A}, \mathcal{B})$ if they are orthogonal to each other with respect to the first extension bi-functor $\operatorname{Ext}_{\mathcal{C}}^{1}(-,-)$, i.e., the following equalities are satisfied:

- $\mathcal{A}={ }^{\perp} \mathcal{B}:=\left\{X \in \mathrm{Ob}(\mathcal{C}) / \operatorname{Ext}_{\mathcal{C}}^{1}(X, B)=0\right.$ for every $\left.B \in \mathcal{B}\right\}$.

- $\mathcal{B}=\mathcal{A}^{\perp}:=\left\{X \in \mathrm{Ob}(\mathcal{C}) / \operatorname{Ext}_{\mathcal{C}}^{1}(A, X)=0\right.$ for every $\left.A \in \mathcal{A}\right\}$.

We are interested in a special class of cotorsion pairs $(\mathcal{A}, \mathcal{B})$ from which it is possible to obtain pre-covers and pre-envelopes by the left and right halves of $(\mathcal{A}, \mathcal{B})$, respectively. A cotorsion pair $(\mathcal{A}, \mathcal{B})$ in $\mathcal{C}$ is complete if, for every object $X$ of $\mathcal{C}$, there exist two short exact sequences

$$
0 \longrightarrow B \longrightarrow A \longrightarrow X \longrightarrow 0
$$

and

$$
0 \longrightarrow X \longrightarrow B^{\prime} \longrightarrow A^{\prime} \longrightarrow 0
$$

where $A, A^{\prime} \in \mathcal{A}$ and $B, B^{\prime} \in \mathcal{B}$. The map $A \rightarrow X$ is called a special $\mathcal{A}$-pre-cover and $X \rightarrow B^{\prime}$ a special $\mathcal{B}$-pre-envelope.

Two cotorsion pairs $\left(\mathcal{A}, \mathcal{B}^{\prime}\right)$ and $\left(\mathcal{A}^{\prime}, \mathcal{B}\right)$ are compatible if there exists a class of objects $\mathcal{W}$ in $\mathcal{C}$ such that $\mathcal{A}^{\prime}=\mathcal{A} \cap \mathcal{W}$ and $\mathcal{B}^{\prime}=\mathcal{B} \cap \mathcal{W}$.

A class of objects $\mathcal{A}$ in $\mathcal{C}$ is said to be thick if it is closed under retracts, and if for every exact sequence,

$$
0 \longrightarrow A^{\prime} \longrightarrow A \longrightarrow A^{\prime \prime} \longrightarrow 0,
$$


two out of three of the terms $A^{\prime}, A$ and $A^{\prime \prime}$ are in $\mathcal{A}$, then so is the third.

Theorem 2.1 (Hovey's correspondence, [14, Theorem 2.2]). Let $\mathcal{C}$ be a bi-complete Abelian category. If $(\mathcal{A}, \mathcal{B} \cap \mathcal{W})$ and $(\mathcal{A} \cap \mathcal{W}, \mathcal{B})$ are complete cotorsion pairs in $\mathcal{C}$ and the class $\mathcal{W}$ is thick, then there exists a unique Abelian model structure on $\mathcal{C}$ such that $\mathcal{A}$ is the class of cofibrant objects. $\mathcal{B}$ is the class of fibrant objects and $\mathcal{W}$ is the class of trivial objects.

Conversely, if $\mathcal{C}$ is equipped with an Abelian model structure and $\mathcal{A}$, $\mathcal{B}$ and $\mathcal{W}$ denote the classes of cofibrant, fibrant and trivial objects, respectively, then $(\mathcal{A} \cap \mathcal{W}, \mathcal{B})$ and $(\mathcal{A}, \mathcal{B} \cap \mathcal{W})$ are complete cotorsion pairs.

Roughly speaking, a model structure on a bi-complete category $\mathcal{C}$ is given by three classes of morphisms of $\mathcal{C}$, called cofibrations, fibrations and weak equivalences, such that it is possible to use homotopy theory in $\mathcal{C}$. A trivial or acyclic cofibration is a map which is a cofibration and a weak equivalence. Trivial fibrations are defined similarly. We do not recall the axioms defining a model structure, but a good introduction to this notion along with very detailed examples is in [15].

If we are given a model structure on an Abelian category, $\mathcal{C}$, an object $X$ of $\mathcal{C}$ is said to be cofibrant (respectively, fibrant) if the map $0 \rightarrow X$ is a cofibration (respectively, if $X \rightarrow 0$ is a fibration). If $0 \rightarrow X$ is a weak equivalence, then $X$ is said to be trivial or acyclic.

A model structure on a bi-complete Abelian category $\mathcal{C}$ is said to be Abelian if the following two conditions are satisfied:

- A map $f$ is a (trivial) cofibration if, and only if, $f$ is a monomorphism and CoKer $(f)$ is cofibrant (and trivial).

- A map $g$ is a (trivial) fibration if, and only if, $g$ is an epimorphism and $\operatorname{Ker}(g)$ is fibrant (and trivial).

Eklof and Trlifaj's theorem is probably one of the most effective methods for obtaining complete cotorsion pairs. It states that, if $\mathcal{S}$ is a set of objects in a Grothendieck category, then the cotorsion pair $\left({ }^{\perp}\left(\mathcal{S}^{\perp}\right), \mathcal{S}^{\perp}\right.$ ) is complete (this theorem was originally proven in $[\mathbf{6}$, 
Theorem 10] for the category of modules, but it is also valid in every Grothendieck category, as specified in [11, Remark 3.2.2]).

If $(\mathcal{A}, \mathcal{B})$ is a cotorsion pair such that $\mathcal{B}=\mathcal{S}^{\perp}$ for some set $\mathcal{S}$, then $(\mathcal{A}, \mathcal{B})$ is said to be cogenerated by $\mathcal{S}$. In this work, every time we want to show that a cotorsion pair $(\mathcal{A}, \mathcal{B})$ is complete, we shall find a set $\mathcal{S}$ such that every object in $\mathcal{A}$ is $\mathcal{S}$-filtered. Under these conditions, the pair $(\mathcal{A}, \mathcal{B})$ is cogenerated by $\mathcal{S}$.

A transfinite composition in a cocomplete Abelian category $\mathcal{C}$ is a morphism of the form $f: F_{0} \rightarrow \operatorname{CoLim}_{\alpha<\lambda}\left(F_{\alpha}\right)$, where $F:[\lambda] \rightarrow \mathcal{C}$ is a colimit preserving functor and $\lambda$ is an ordinal. The morphism $f$ is also known as the transfinite composition of the morphisms $F_{\alpha} \rightarrow F_{\alpha+1}$ for every $\alpha+1<\lambda$. If, in addition, all the morphisms $F_{\alpha} \rightarrow F_{\alpha+1}$ are monic with cokernel in some class $\mathcal{S}, F_{0} \stackrel{f}{\rightarrow} \operatorname{CoLim}_{\alpha<\lambda}\left(F_{\alpha}\right)$ is called a transfinite extension of $F_{0}$ by $\mathcal{S}$. If $F_{0} \in \mathcal{S}$ as well, the colimit $\operatorname{CoLim}_{\alpha<\lambda}\left(F_{\alpha}\right)$ is called a transfinite extension of $\mathcal{S}$.

If $X$ is an object of $\mathcal{C}$ such that $X=\operatorname{CoLim}_{\alpha<\lambda}\left(F_{\alpha}\right)$ is a transfinite extension of $\mathcal{S}, X$ is said to be $\mathcal{S}$-filtered and the family $\left(F_{\alpha}: \alpha<\lambda\right)$ an $\mathcal{S}$-filtration.

Definition 2.2. Let $\mathcal{A}$ be a class of objects in an Abelian category $\mathcal{C}$. We shall say that an object $X$ has a left $\mathcal{A}$-resolution if there exists an exact sequence

$$
\cdots \longrightarrow A_{1} \longrightarrow A_{0} \longrightarrow X \longrightarrow 0,
$$

where $A_{k} \in \mathcal{A}$ for every $k \in \mathbb{Z}_{\geq 0}$. In this sense, we shall say that $X$ is a left $n$-A-object if it has a finite left $\mathcal{A}$-resolution of length $n$, i.e., an exact sequence as above where $A_{k}=0$ for every $k>n$. Right $\mathcal{A}$-resolutions and right $n$ - $\mathcal{A}$-objects are defined dually.

We recall that a cotorsion pair $(\mathcal{A}, \mathcal{B})$ in $\mathcal{C}$ is hereditary if $\operatorname{Ext}_{\mathcal{C}}^{i}(A, B)$ is null for every $A \in \mathcal{A}, B \in \mathcal{B}$ and $i>1$. The following result is easy to prove.

Lemma 2.3. Let $(\mathcal{A}, \mathcal{B})$ be a hereditary cotorsion pair in an Abelian category $\mathcal{C}$. Then the classes of left $n$-A-objects and right $n$ - $\mathcal{B}$-objects are both closed under extensions. 
The following lemma is proven in the category of left $R$-modules in [11], but the arguments appearing there carry over to every Grothendieck category.

Lemma 2.4 (Eklof [11, Lemma 3.1.2]). Let $X$ and $Y$ be two objects of a cocomplete Abelian category $\mathcal{C}$, and suppose $X=\operatorname{CoLim}_{\alpha<\lambda}\left(X_{\alpha}\right)$, where $\left(X_{\alpha}: \alpha<\lambda\right)$ is a transfinite extension of ${ }^{\perp}\{Y\}$. Then $X \in{ }^{\perp}\{Y\}$.

As a consequence, we have the following proposition.

Proposition 2.5. Let $(\mathcal{A}, \mathcal{B})$ be a cotorsion pair in an Abelian category $\mathcal{C}$. If $\mathcal{S} \subseteq \mathcal{A}$ is a set of objects in $\mathcal{C}$ such that every object of $\mathcal{A}$ is $\mathcal{S}$ filtered, then $(\mathcal{A}, \mathcal{B})$ is cogenerated by $\mathcal{S}$.

3. Cotorsion pairs from projective dimensions of left modules over a ringoid. In this section, we shall study some facts concerning the projective dimension of objects in an Abelian category $\mathcal{C}$. We shall focus on the particular case where $\mathcal{C}$ is the category $\operatorname{Mod}(\Re)$ of modules over a ringoid $\mathfrak{R}$, for which we prove the class of objects with projective dimension bounded by some $n \geq 0$ is the left half of a cotorsion pair cogenerated by a set. This was initially proven by Aldrich, et al., in [2] in the case $\mathcal{C}$ is the category of left $R$-modules ${ }_{R}$ Mod. We provide some modifications to their arguments to generalize their results to $\operatorname{Mod}(\Re)$. The advantage in doing this lies in the fact that both ${ }_{R}$ Mod and the category $\mathbf{C h}\left({ }_{R} \mathbf{M o d}\right)$ of complexes of the left $R$-modules are particular examples of categories of modules over a ringoid.

We recall some notation in the category $\mathbf{C h}(\mathcal{C})$ of complexes over an Abelian category $\mathcal{C}$. Given a chain complex $X=\left(X_{m}\right)_{m \in \mathbb{Z}}$ with boundary or differential maps $\partial_{m}^{X}: X_{m} \rightarrow X_{m-1}$, the object $Z_{m}(X):=$ $\operatorname{Ker}\left(\partial_{m}^{X}\right)$ is called the $m$-cycle of $X$, and $B_{m}(X):=\operatorname{Im}\left(\partial_{m+1}^{X}\right)$ the $m$ boundary of $X$.

Example 3.1. Let $C \in \mathrm{Ob}(\mathcal{C})$. The $m$ th sphere complex centered at $C$, denoted $S^{m}(C)$, is defined by $\left(S^{m}(C)\right)_{m}:=C$ and $\left(S^{m}(C)\right)_{k}:=0$ if $k \neq m$. The $m$ th disk complex centered at $C$, denoted $D^{m}(C)$, is 
defined by $\left(D^{m}(C)\right)_{k}:=C$ if $k=m$ or $m-1$, and 0 otherwise, where the only non-zero boundary map $\partial_{m}^{D^{m}(C)}$ is the identity id ${ }_{C}$.

From now on, left $n-\mathcal{P}_{0}(\mathcal{C})$-objects and right $n$ - $\mathcal{I}_{0}(\mathcal{C})$-objects shall be called $n$-projective and $n$-injective objects, respectively. Let $X$ be an object in an Abelian category $\mathcal{C}$. Let $\operatorname{pd}(X)$ and id $(X)$ denote the projective and injective dimension of $X$, respectively. Note that $X$ is $n$-projective (respectively, $n$-injective) if, and only if, $\operatorname{pd}(X) \leq n$ (respectively, id $(X) \leq n$ ). We shall denote by $\mathcal{P}_{n}(\mathcal{C})$ and $\mathcal{I}_{n}(\mathcal{C})$ the classes of $n$-projective and $n$-injective objects of $\mathcal{C}$, respectively.

Example 3.2 ([2, Theorem 4.2]). Let $R$ be an associative ring with unity and $\kappa$ an infinite cardinal such that $\kappa \geq \operatorname{Card}(R)$. The class $\mathcal{P}_{n}:=\mathcal{P}_{n}\left({ }_{R} \mathbf{M o d}\right)$ of $n$-projective modules is the left half of a cotorsion pair $\left(\mathcal{P}_{n},\left(\mathcal{P}_{n}\right)^{\perp}\right)$ cogenerated by a set of representatives by the class

$$
\left(\mathcal{P}_{n}\right)^{\leq \kappa}:=\left\{M \in \mathcal{P}_{n}: \operatorname{Card}(M) \leq \kappa\right\} .
$$

Using induction and the fact that the class of exact chain complexes is thick, the following result follows.

Lemma 3.3. Let $\mathcal{C}$ be an Abelian category.

(i) If

$$
0 \longrightarrow A_{n} \longrightarrow \cdots \longrightarrow A_{0} \longrightarrow X \longrightarrow 0
$$

is an exact sequence in $\mathbf{C h}(\mathcal{C})$ such that $A_{i}$ is exact for every $0 \leq i \leq n$, then so is $X$.

(ii) If

$$
0 \longrightarrow Y \longrightarrow B^{0} \longrightarrow \cdots \longrightarrow B^{n} \longrightarrow 0
$$

is an exact sequence in $\mathbf{C h}(\mathcal{C})$ such that $B^{i}$ is exact for every $0 \leq i \leq n$, then so is $Y$.

Lemma 3.4. Consider a short exact sequence

$$
0 \longrightarrow Y \stackrel{f}{\longrightarrow} Z \stackrel{g}{\longrightarrow} X \longrightarrow 0
$$

of chain complexes over $\mathcal{C}$. 
(i) The sequence

$$
0 \longrightarrow Z_{m}(Y) \longrightarrow Z_{m}(Z) \longrightarrow Z_{m}(X) \longrightarrow 0
$$

is exact if $Y$ is an exact complex.

(ii) The sequence

$$
0 \longrightarrow \frac{Y_{m}}{B_{m}(Y)} \longrightarrow \frac{Z_{m}}{B_{m}(Z)} \longrightarrow \frac{X_{m}}{B_{m}(X)} \longrightarrow 0
$$

is exact if $X$ is an exact complex.

Proof. We only show the first statement for ${ }_{R}$ Mod, by [19, Theorem 3, page 204]. Let $Z_{m}(f): Z_{m}(Y) \rightarrow Z_{m}(Z)$ be the homomorphism induced by the universal property of kernels, given by $y \mapsto f_{m}(y)$ for every $y \in Z_{m}(Y)$. The homomorphism $Z_{m}(g): Z_{m}(Y) \rightarrow Z_{m}(X)$ is defined similarly. It is easy to check that $Z_{m}(f)$ is monic and that $\operatorname{Ker}\left(Z_{m}(g)\right)=\operatorname{Im}\left(Z_{m}(f)\right)$. These facts do not depend on the exactness of $Y$. Let $x \in Z_{m}(X)$. There exists $z \in Z_{m}$ such that $x=g_{m}(z)$. We have $g_{m-1} \circ \partial_{m}^{Z}(z)=\partial_{m}^{X} \circ g_{m}(z)=0$. Since the sequence

$$
0 \longrightarrow Y_{m-1} \longrightarrow Z_{m-1} \longrightarrow X_{m-1} \longrightarrow 0
$$

is exact, there exists $y \in Y_{m-1}$ such that $\partial_{m}^{Z}(z)=f_{m-1}(y)$. Then $f_{m-2} \circ \partial_{m-1}^{Y}(y)=\partial_{m-1}^{Z} \circ f_{m-1}(y)=0$, and so $\partial_{m-1}^{Y}(y)=0$ since $f_{m-2}$ is monic. By the exactness of $Y$ there exists $y^{\prime} \in Y_{m}$ such that $y=\partial_{m}^{Y}\left(y^{\prime}\right)$. Hence, $\partial_{m}^{Z}\left(z-f_{m}\left(y^{\prime}\right)\right)=0$ and $g_{m}\left(z-f_{m}\left(y^{\prime}\right)\right)=x$.

Using Lemma 3.4 along with the induction principle, we obtain the following result.

Lemma 3.5. Let

$$
0 \longrightarrow A_{n} \stackrel{f_{n}}{\longrightarrow} A_{n-1} \longrightarrow \cdots \longrightarrow A_{1} \stackrel{f_{1}}{\longrightarrow} A_{0} \longrightarrow 0
$$

be an exact sequence in $\mathbf{C h}(\mathcal{C})$ of exact chain complexes. Then for every $m \in \mathbb{Z}$, the $m$-cycles $Z_{m}\left(A_{i}\right)$ form the following exact sequence in $\mathcal{C}$ :

$0 \longrightarrow Z_{m}\left(A_{n}\right) \longrightarrow Z_{m}\left(A_{n-1}\right) \longrightarrow \cdots \longrightarrow Z_{m}\left(A_{1}\right) \longrightarrow Z_{m}\left(A_{0}\right) \longrightarrow 0$.

Statement (ii) of the next proposition is proven in [8, Theorem 3.1.3]. We give here a different argument. 
Proposition 3.6. Let $\mathcal{C}$ be an Abelian category and $n$ a positive integer.

(i) Assume $\mathcal{C}$ has enough projective objects. A chain complex $X$ is $n$-projective if, and only if, $X$ is exact and each $m$-cycle is an $n$-projective object of $\mathcal{C}$.

(ii) Assume $\mathcal{C}$ has enough injective objects. A chain complex $Y$ is $n$-injective if, and only if, $Y$ is exact and each $m$-cycle is an $n$ injective object of $\mathcal{C}$.

Proof. Let $X$ be an exact complex with $n$-projective cycles. Consider a partial projective resolution

$$
0 \longrightarrow K \longrightarrow P_{n-1} \longrightarrow \cdots \longrightarrow P_{0} \longrightarrow X \longrightarrow 0 .
$$

Note that $K$ is exact by Lemma 3.3. Notice also that $Z_{m}\left(P_{i}\right)$ is projective for every $0 \leq i \leq n-1$ and every $m \in \mathbb{Z}$. It follows by Lemma 3.5 that $Z_{m}(K) \in \Omega^{n}\left(Z_{m}(X)\right.$ ) (where $\Omega^{n}\left(Z_{m}(X)\right.$ ) denotes the class of $n$-syzygies of $\left.Z_{m}(X)\right)$. Hence, $Z_{m}(K) \in \mathcal{P}_{0}(\mathcal{C})$ since $Z_{m}(X)$ is $n$-projective. The converse follows similarly.

We shall see that the class $\mathcal{P}_{n}\left(\mathbf{C h}\left({ }_{R} \mathbf{M o d}\right)\right)$ of $n$-projective chain complexes is the left half of a complete cotorsion pair. This, along with Example 3.2, is a consequence of a more general result in the category $\operatorname{Mod}(\mathfrak{R})$ of left modules over a ringoid $\mathfrak{R}$. We begin by studying the projective dimension of objects in this category.

A small pre-additive category $\mathfrak{R}$ is called ringoid or ring with many objects. Then we have a composition law

$$
\begin{aligned}
\operatorname{Hom}_{\mathfrak{R}}(b, c) \otimes \operatorname{Hom}_{\mathfrak{R}}(a, b) & \longrightarrow \operatorname{Hom}_{\mathfrak{R}}(a, c) \\
y \otimes x & \longmapsto y \circ x
\end{aligned}
$$

for every $a, b, c \in \mathrm{Ob}(\mathfrak{R})$, and a unit id $a \in \operatorname{Hom}_{\mathfrak{R}}(a, a)$ for every $a \in$ $\mathrm{Ob}(\mathfrak{R})$. The composition law defines a ring structure on $\operatorname{Hom}_{\mathfrak{R}}(a, a)$ for every $a \in \mathrm{Ob}(\mathfrak{R})$. Moreover, the Abelian group $\operatorname{Hom}_{\mathfrak{R}}(a, b)$ has the structure of a bi-module, with a left action by $\operatorname{Hom}_{\mathfrak{R}}(b, b)$ and a right action by $\operatorname{Hom}_{\Re}(a, a)$.

\section{Example 3.7.}

(i) Every ring $R$ can be regarded as a ringoid $\mathfrak{R}$ having a single object $\star$ if we put $\operatorname{Hom}_{\mathfrak{R}}(\star, \star)=R$. 
(ii) We shall denote by $\mathfrak{S}$ the ringoid generated by the following infinite graph together with the relation $\partial_{n} \circ \partial_{n+1}=0$ for $n \in \mathbb{Z}$.

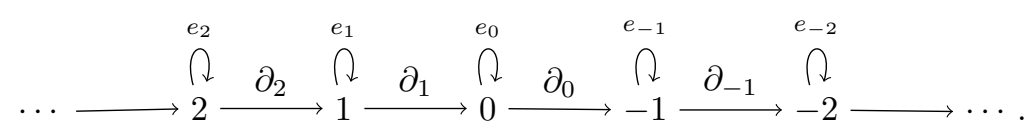

We have $\mathrm{Ob}(\mathfrak{S})=\mathbb{Z}$ and

$$
\operatorname{Hom}_{\mathfrak{S}}(i, j)= \begin{cases}\left\langle e_{i}\right\rangle:=\left\{m \cdot e_{i}: m \in \mathbb{Z}\right\} & \text { if } j=i \\ \left\langle\partial_{i}\right\rangle:=\left\{m \cdot \partial_{i}: m \in \mathbb{Z}\right\} & \text { if } j=i+1 \\ 0 & \text { otherwise }\end{cases}
$$

A (left) module over $\mathfrak{R}$ is an additive functor $M: \mathfrak{R} \rightarrow \mathbf{A b}$, where $\mathbf{A b}$ is the category of Abelian groups. A map of (left) $\mathfrak{R}$-modules is a natural transformation $M \rightarrow N$.

Example 3.8. Given a ringoid $\mathfrak{R}$ and $a \in \mathrm{Ob}(\mathfrak{R})$, the covariant functor $\operatorname{Hom}_{\mathfrak{R}}(a,-): \mathfrak{R} \rightarrow \mathbf{A b}$ is a (left) $\mathfrak{R}$-module.

The category $\operatorname{Mod}(\mathfrak{R})$ of left modules over $\mathfrak{R}$ is defined as the category $[\mathfrak{R}, \mathbf{A} \mathbf{b}]$ of additive functors $\mathfrak{R} \rightarrow \mathbf{A b}$. Note that $\operatorname{Mod}(\mathfrak{R})$ is Abelian and bi-complete since $\mathbf{A b}$ is.

Example 3.9 (Specific examples of $\operatorname{Mod}(\mathfrak{R}))$.

(i) If $\mathfrak{R}$ is the ringoid of Example 3.7 (i), then $\operatorname{Mod}(\mathfrak{R})$ is the category ${ }_{R}$ Mod of left $R$-modules.

(ii) Recall that the tensor product $\mathcal{C} \otimes \mathcal{D}$ of two pre-additive categories $\mathcal{C}$ and $\mathcal{D}$ is the pre-additive category defined by putting $\mathrm{Ob}(\mathcal{C} \otimes \mathcal{D})=\operatorname{Ob}(\mathcal{C}) \times \mathrm{Ob}(\mathcal{D})$ and $\operatorname{Hom}_{\mathcal{C} \otimes \mathcal{D}}\left((C, D),\left(C^{\prime}, D^{\prime}\right)\right)=$ $\operatorname{Hom}_{\mathcal{C}}\left(C, C^{\prime}\right) \otimes \operatorname{Hom}_{\mathcal{D}}\left(D, D^{\prime}\right)$. If $\mathcal{C}, \mathcal{D}$ and $\mathcal{E}$ are pre-additive categories, then we have a canonical isomorphism of pre-additive categories $[\mathcal{C} \otimes \mathcal{D}, \mathcal{E}] \simeq[\mathcal{C},[\mathcal{D}, \mathcal{E}]]$. In particular, if $\mathcal{K}$ and $\mathfrak{R}$ are ringoids, then $[\mathcal{K}, \operatorname{Mod}(\mathfrak{R})]=[\mathcal{K},[\mathfrak{R}, \mathbf{A b}]] \simeq[\mathcal{K} \otimes \mathfrak{R}, \mathbf{A b}]=$ $\operatorname{Mod}(\mathcal{K} \otimes \mathfrak{R})$. If we consider a ring $R$ and the ringoid $\mathfrak{S}$ defined in Example 3.7 (ii), then we have an isomorphism $[\mathfrak{S}, \operatorname{Mod}(R)] \cong$ $\operatorname{Mod}(\mathfrak{S} \otimes R)$ of additive categories. This means that a chain 
complex of $R$-modules is a module over the ringoid $\mathfrak{S} \otimes R$, i.e., $\mathbf{C h}\left({ }_{R} \operatorname{Mod}\right) \cong \operatorname{Mod}(\mathfrak{S} \otimes R)$.

Remark 3.10. A sequence

$$
\cdots \longrightarrow M_{1} \longrightarrow M_{0} \longrightarrow M_{-1} \longrightarrow \cdots
$$

of left $\mathfrak{R}$-modules is exact if, for every $a \in \mathrm{Ob}(\mathfrak{R})$, the sequence of Abelian groups

$$
\cdots \longrightarrow M_{1}(a) \longrightarrow M_{0}(a) \longrightarrow M_{-1}(a) \longrightarrow \cdots
$$

is exact.

Definition 3.11. We shall say that an element $x \in M(a)$ is homogenous of grade $a$, and we shall write $a=|x|$.

If $M$ is a left module over $\mathfrak{R}$, then the map

$$
\operatorname{Hom}_{\mathfrak{R}}(a, b) \longrightarrow \operatorname{Hom}_{\mathbf{A b}}(M(a), M(b))
$$

of Abelian groups defined by $M$ induces a multiplication

$$
\begin{aligned}
\operatorname{Hom}_{\mathfrak{R}}(a, b) \otimes M(a) & \longrightarrow M(b) \\
(r, x) & \longmapsto r \cdot x:=M(r)(x),
\end{aligned}
$$

for every $a, b \in \mathrm{Ob}(\mathfrak{R})$. The product of $r \in \operatorname{Hom}_{\mathfrak{R}}(a, b)$ by $x \in M(a)$ is an element $r \cdot x \in M(b)$.

Definition 3.12. We shall say that a linear combination of homogenous elements $y=\sum_{i \in I} r_{i} \cdot x_{i}$ is admissible if $y$ is homogenous and $r_{i} \in \operatorname{Hom}_{\mathfrak{R}}\left(\left|x_{i}\right|,|y|\right)$ for every $i \in I$. We shall accept infinite combinations in the case where $r_{i}=0$ for all but finitely many $i \in I$.

Definition 3.13. If $M$ is a left $\mathfrak{R}$-module, we shall say that a family $N=\{N(a): a \in \mathrm{Ob}(\mathfrak{R})\}$ of subgroups $N(a) \subseteq M(a)$ is a submodule of $M$ if $x \in N(a)$ implies $r \cdot x \in N(b)$ for every $r \in \operatorname{Hom}_{\mathfrak{R}}(a, b)$.

Remark 3.14. Note that the family $\{N(a): a \in \mathrm{Ob}(\mathfrak{R})\}$ in the previous definition defines a functor $N: \mathfrak{R} \rightarrow \mathbf{A b}$, where $N(r)$ is the restriction $\left.M(r)\right|_{N(a)}$ for every map $r: a \rightarrow b$. Conversely, if $N$ is a sub-functor of $M$, then $\{N(a): a \in \mathrm{Ob}(\Re)\}$ is a submodule of $M$. 
We want to construct, for every $n$-projective left $\mathfrak{R}$-module, a transfinite extension of "small" $n$-projective $\mathfrak{R}$-modules. The construction of this transfinite extension in the case $\mathcal{C}={ }_{R}$ Mod is based on a method, probably first introduced by Aldrich, et al., in [2], known as the zigzag procedure. We shall explain how to adapt this procedure to the category $\operatorname{Mod}(\mathfrak{R})$.

We need to introduce some notation and recall the notion of free $\mathfrak{R}$-modules.

Definition 3.15. If $M$ is an $\Re$-module, we shall say that a submodule $N \subseteq M$ is generated by a family $\left\{x_{i}\right\}_{i \in I}$ of homogenous elements if $N$ is the smallest submodule of $M$ which contains all the elements $x_{i}$.

Let $N \subseteq M$ be a submodule generated by a family $\left\{x_{i}\right\}_{i \in I}$ of homogenous elements of $M$. Then an element $x \in M(a)$ belongs to $N(a)$ if and only if it is an admissible linear combination

$$
x=\sum_{i \in I} r_{i} \cdot x_{i}
$$

(where $r_{i}=0$ for all but finitely many $i \in I$ ).

Definition 3.16. We shall say that a family $\left\{x_{i}\right\}_{i \in I}$ of homogenous elements of $M$ is a basis of $M$ if every homogenous element $x \in M$ can be written uniquely as an admissible linear combination

$$
x=\sum_{i \in I} r_{i} \cdot x_{i} .
$$

We shall say that $M$ is free if it admits a basis.

If $\mathfrak{R}$ is a ringoid and $M$ is a left $\mathfrak{R}$-module, then it follows from Yoneda's lemma that, for every $a \in \mathrm{Ob}(\Re)$ and every $x \in M(a)$, there is a unique map of $\Re$-modules $\alpha: \operatorname{Hom}_{\mathfrak{R}}(a,-) \rightarrow M$ such that $x=\alpha_{a}\left(\mathrm{id}_{a}\right)$. More generally, if $\left\{a_{i}\right\}_{i \in I}$ is a family of objects of $\mathfrak{R}$, let us put

$$
\left\langle a_{i}: i \in I\right\rangle:=\bigoplus_{i \in I} \operatorname{Hom}_{\Re}\left(a_{i},-\right)
$$


and $[i]:=\left(u_{i}\right)_{a_{i}}\left(\operatorname{id}_{a_{i}}\right)$, where $u_{i}: \operatorname{Hom}_{\mathfrak{R}}\left(a_{i},-\right) \rightarrow\left\langle a_{i}: i \in I\right\rangle$ is the inclusion. Consider a family of elements $\left\{x_{i}\right\}_{i \in I}$ in $\prod_{i \in I} M\left(a_{i}\right)$. Then, for each $i \in I$, we can write $x_{i}=\left(\alpha^{i}\right)_{a_{i}}\left(\mathrm{id}_{a_{i}}\right)$, where $\alpha^{i}$ is a map $\operatorname{Hom}_{\mathfrak{R}}\left(a_{i},-\right) \rightarrow M$ of left $\mathfrak{R}$-modules. Since $\operatorname{Mod}(\mathfrak{R})$ is cocomplete, there exists a unique map $f:\left\langle a_{i}: i \in I\right\rangle \rightarrow M$ such that the following triangle commutes:

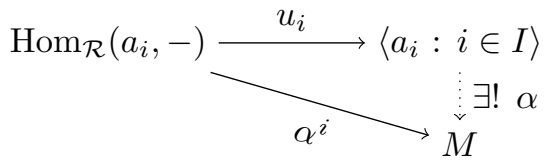

Note that $\alpha_{a_{i}}([i])=\alpha_{a_{i}} \circ\left(u_{i}\right)_{a_{i}}\left(\operatorname{id}_{a_{i}}\right)=\left(\alpha^{i}\right)_{a_{i}}\left(\operatorname{id}_{a_{i}}\right)=x_{i}$ for every $i \in I$. It follows that the $\mathfrak{R}$-module $\left\langle a_{i}: i \in I\right\rangle$ is freely generated by the elements $[i]$ of grade $a_{i}$ for $i \in I$.

Definition 3.17. The family $\left\{a_{i}\right\}_{i \in I}$ defines a map $|-|: I \rightarrow \mathrm{Ob}(\mathfrak{R})$ if we put $|i|=a_{i}$ for $i \in I$. We shall say that the set $I$ equipped with the map $|-|: I \rightarrow \mathrm{Ob}(\mathfrak{R})$ is $\mathfrak{R}$-graded.

If $I$ is an $\mathfrak{R}$-graded set, then the $\mathfrak{R}$-module $\langle I\rangle=\bigoplus_{i \in I} \operatorname{Hom}_{\mathfrak{R}}(|i|,-)$ is freely generated by elements $[i]$ of grade $|i|$ for $i \in I$.

Proposition 3.18. An $\mathfrak{R}$-module $M$ is free if, and only if, it is isomorphic to a coproduct of $\mathfrak{R}$-modules $\operatorname{Hom}_{\mathfrak{R}}\left(a_{i},-\right)$ for a family $\left\{a_{i}\right\}_{i \in I}$ of objects of $\mathfrak{R}$,

$$
M \cong \bigoplus_{i \in I} \operatorname{Hom}_{\mathfrak{R}}\left(a_{i},-\right) .
$$

Proof. The implication $(\Leftarrow)$ follows by the comments above. Now suppose $M$ is a free left module over $\mathfrak{R}$ admitting a basis $\left\{x_{i}\right\}_{i \in I}$. Consider the natural transformation $\alpha:\left\langle a_{i}: i \in I\right\rangle>\rightarrow M$ given above, where $x_{i} \in M\left(a_{i}\right)$. We check that $\alpha$ is a natural isomorphism, i.e., $\alpha_{b}: \bigoplus_{i \in I} \operatorname{Hom}_{\mathfrak{R}}\left(a_{i}, b\right) \rightarrow M(b)$ is an isomorphism for every $b \in \mathrm{Ob}(\mathfrak{R})$. Let $x \in M(b)$. We can write $x$ as a unique admissible linear combination $x=\sum_{i \in I} r_{i} \cdot x_{i}$, where $r_{i} \in \operatorname{Hom}_{\mathfrak{R}}\left(a_{i}, b\right)$ for every $i \in I$. Since $\alpha$ is a natural transformation, we have that 


$$
\begin{aligned}
M\left(r_{i}\right) \circ \alpha_{a_{i}}=\alpha_{b} \circ & \left\langle a_{i}: i \in I\right\rangle\left(r_{i}\right) . \text { Then, } \\
x & =\sum_{i \in I} M\left(r_{i}\right)\left(x_{i}\right)=\sum_{i \in I} M\left(r_{i}\right)\left(\alpha_{a_{i}}([i])\right) \\
& =\sum_{i \in I} \alpha_{b} \circ\left\langle a_{i}: i \in I\right\rangle\left(r_{i}\right)([i]) \\
& =\sum_{i \in I} \alpha_{b}\left(r_{i} \cdot[i]\right)=\alpha_{b}\left(\sum_{i \in I} r_{i} \cdot[i]\right),
\end{aligned}
$$

where $\sum_{i \in I} r_{i} \cdot[i]$ is unique.

Corollary 3.19. Every free (left) $\mathfrak{R}$-module is projective.

Proof. Since the direct sum of projective objects is projective, by the previous proposition it suffices to show that $\operatorname{Hom}_{\mathfrak{R}}(a,-)$ is projective in $\operatorname{Mod}(\mathfrak{R})$, i.e., that the functor $\operatorname{Hom}_{\operatorname{Mod}(\mathfrak{R})}\left(\operatorname{Hom}_{\mathfrak{R}}(a,-),-\right)$ : $\operatorname{Mod}(\Re) \rightarrow \mathbf{A b}$ is exact. Suppose we are given a short exact sequence $0 \rightarrow M^{\prime} \rightarrow M \rightarrow M^{\prime \prime} \rightarrow 0$ in $\operatorname{Mod}(\Re)$. Then the sequence:

$$
\begin{aligned}
0 \longrightarrow \operatorname{Hom}_{\operatorname{Mod}(\mathfrak{R})}\left(\operatorname{Hom}_{\mathfrak{R}}(a,-), M^{\prime}\right) \longrightarrow \operatorname{Hom}_{\operatorname{Mod}(\Re)}\left(\operatorname{Hom}_{\mathfrak{R}}(a,-), M\right) \\
\longrightarrow \operatorname{Hom}_{\operatorname{Mod}(\mathfrak{R})}\left(\operatorname{Hom}_{\mathfrak{R}}(a,-), M^{\prime \prime}\right) \longrightarrow 0
\end{aligned}
$$

is exact since it is isomorphic to

$$
0 \longrightarrow M^{\prime}(a) \longrightarrow M(a) \longrightarrow M^{\prime \prime}(a) \longrightarrow 0,
$$

by Yoneda's lemma.

Proposition 3.20 (Eilenberg's trick for modules over a ringoid). For every projective $\mathfrak{R}$-module $P$, there exists a free $\mathfrak{R}$-module $F$ together with an isomorphism $P \oplus F \cong F$.

As a consequence of the Eilenberg's trick, we have:

Corollary 3.21. Every $n$-projective (left) $\mathfrak{R}$-module has a left free resolution of length $n$. 
Definition 3.22. Let $\kappa$ be an infinite regular cardinal strictly greater than the cardinality of $\operatorname{Hom}_{\mathfrak{R}}(a, b)$ for every $a, b \in \mathrm{Ob}(\mathfrak{R})$. We shall say that an $\Re$-module $M$ is $\kappa$-small if Card $(M(a)) \leq \kappa$, for every $a \in \mathrm{Ob}(\mathfrak{R})$.

Remark 3.23 ( $\kappa$-small modules and complexes). Let $\mathfrak{R}$ be a ringoid and $\kappa$ an infinite regular cardinal such that $\kappa>\operatorname{Card}\left(\operatorname{Hom}_{\mathfrak{R}}(a, b)\right)$ for every $a, b \in \mathrm{Ob}(\mathfrak{R})$. Given a class $\mathcal{X}$ of left modules over $\mathfrak{R}$, we shall denote by $\mathcal{X} \leq \kappa$ the class of $\kappa$-small left modules in $\mathcal{X}$.

(i) If $\mathfrak{R}$ is the ringoid of Example 3.7 (i), then we have that $\kappa>$ Card $(R)$ and that a left $R$-module $M$ is $\kappa$-small if, and only if, $\operatorname{Card}(M) \leq \kappa$.

(ii) We know $\mathbf{C h}\left({ }_{R} \mathbf{M o d}\right)$ is equivalent to the category of left modules over the ringoid $\mathfrak{S} \otimes R$, with $\mathfrak{S}$ as in Example 3.7 (ii). It is not hard to see that $\kappa>\operatorname{Card}(R)$ and that the following conditions are equivalent for every chain complex $X$ in $\mathbf{C h}\left({ }_{R} \mathbf{M o d}\right)$ :

(a) $X$ is $\kappa$-small.

(b) $\operatorname{Card}\left(X_{m}\right) \leq \kappa$ for every $m \in \mathbb{Z}$.

(c) $\sum_{m \in \mathbb{Z}} \operatorname{Card}\left(X_{m}\right) \leq \kappa$.

We shall say for the rest of this work that a left $R$-module $M$ is $\kappa$ small if Card $(M) \leq \kappa$, and that a chain complex $X$ in $\mathbf{C h}\left({ }_{R} \mathbf{M o d}\right)$ is $\kappa$-small if each $X_{m}$ is a $\kappa$-small module, where $\kappa$ is an (infinite) regular cardinal satisfying $\kappa>\operatorname{Card}(R)$.

Lemma 3.24 (Generalization of [2, Proposition 4.1]). Let $\kappa$ be an infinite regular cardinal as in Definition 3.22. Let $M$ be an n-projective $\mathfrak{R}$-module. Then for every homogeneous element $x \in M(a)$ there exists a $\kappa$-small submodule $N \hookrightarrow M$ with $x \in N(a)$ such that the $\mathfrak{R}$-modules $N$ and $M / N$ are $n$-projective.

Proof. By Corollary 3.21, we start with a free resolution of $M$, say

$$
0 \longrightarrow\left\langle I_{n}\right\rangle \stackrel{\partial_{n}}{\longrightarrow}\left\langle I_{n-1}\right\rangle \longrightarrow \cdots \longrightarrow\left\langle I_{1}\right\rangle \stackrel{\partial_{1}}{\longrightarrow}\left\langle I_{0}\right\rangle \stackrel{\partial_{0}}{\longrightarrow} M \longrightarrow 0,
$$

where $I_{k}$ is an $\mathfrak{R}$-graded set for every $0 \leq k \leq n$.

The map

$$
\left(\partial_{0}\right)_{a}:\left\langle I_{0}\right\rangle(a)=\bigoplus_{i \in I_{0}} \operatorname{Hom}_{\mathfrak{R}}([i], a) \longrightarrow M(a)
$$


is surjective, so we can find a finite number of maps $r_{i_{1}}:\left[i_{1}\right] \rightarrow$ $a, \ldots, r_{i_{k}}:\left[i_{k}\right] \rightarrow a$ such that $x=\left(\partial_{0}\right)_{a}\left(r_{i_{1}}+\cdots+r_{i_{k}}\right)$. Then $Z_{0}=\left\{i_{1}, \ldots, i_{k}\right\}$ is a finite subset of $I_{0}$ such that $x \in \partial_{0}\left(\left\langle Z_{0}\right\rangle\right)$ (by abuse of notation, this shall mean that $\left.x \in\left(\partial_{0}\right)_{a} \circ\left\langle Z_{0}\right\rangle(a)\right)$. Consider the natural transformation $\left.\partial_{0}\right|_{\left\langle Z_{0}\right\rangle}:\left\langle Z_{0}\right\rangle \rightarrow M$, and let $y \in \operatorname{Ker}\left(\left.\partial_{0}\right|_{\left\langle Z_{0}\right\rangle}\right)$ be of degree $b$. Since $\left\langle I_{1}\right\rangle(b) \rightarrow\left\langle I_{0}\right\rangle(b) \rightarrow M(b)$ is exact, there exists $y^{\prime} \in\left\langle I_{1}\right\rangle(b)$ such that $y=\left(\partial_{1}\right)_{b}\left(y^{\prime}\right)$. We can write $y^{\prime}=\sum_{i \in Z_{1}^{y}} r_{i} \cdot[i]$, where $Z_{1}^{y}$ is a finite subset of $I_{1}$. Let $Z_{1}=\bigsqcup\left\{Z_{1}^{y}: y \in \operatorname{Ker}\left(\left.\partial_{0}\right|_{\left\langle Z_{0}\right\rangle}\right)\right\}$. In order to estimate the number of elements of $Z_{1}^{y}$, note that for each $y \in \operatorname{Ker}\left(\left.\partial_{0}\right|_{\left\langle Z_{0}\right\rangle}\right)$ we have a unique tuple $\left(\rho_{i}: i \in Z_{0}\right)$, with $\rho_{i} \in \operatorname{Hom}_{\mathfrak{R}}([i], b)$. Then we have

$$
\begin{aligned}
\operatorname{Card}\left(Z_{1}\right) & =\sum\left\{\operatorname{Card}\left(Z_{1}^{y}\right): y \in \operatorname{Ker}\left(\left.\partial_{0}\right|_{\left\langle Z_{0}\right\rangle}\right)\right\} \\
& \leq \operatorname{Card}\left(\operatorname{Ker}\left(\left.\partial_{0}\right|_{\left\langle Z_{0}\right\rangle}\right)\right) \text { since each } Z_{1}^{y} \text { is finite, } \\
& \leq \operatorname{Card}\left(\left\{\left(r_{i}: i \in Z_{0}\right): y \in \operatorname{Ker}\left(\left.\partial_{0}\right|_{\left\langle Z_{0}\right\rangle}\right)\right\}\right) \\
& \leq \prod_{i \in Z_{0}} \operatorname{Card}\left(\operatorname{Hom}_{\Re}([i], b)\right) \leq \kappa,
\end{aligned}
$$

and so $Z_{1}$ is a $\kappa$-small subset of $I_{1}$ such that $\partial_{1}\left(\left\langle Z_{1}\right\rangle\right) \supseteq \operatorname{Ker}\left(\left.\partial_{0}\right|_{\left\langle Z_{0}\right\rangle}\right)$, i.e., $\left(\partial_{1}\right)_{b}\left(\left\langle Z_{1}\right\rangle(b)\right) \supseteq \operatorname{Ker}\left(\left.\left(\partial_{0}\right)_{b}\right|_{\left\langle Z_{0}\right\rangle(b)}\right)$ for every $b \in \operatorname{Ob}(\Re)$. In a similar way, we can find a $\kappa$-small subset $Z_{2} \subseteq I_{2}$ such that $\partial_{2}\left(\left\langle Z_{2}\right\rangle\right) \supseteq$ $\operatorname{Ker}\left(\left.\partial_{1}\right|_{\left\langle Z_{1}\right\rangle}\right)$. We keep repeating this procedure until we get a $\kappa$-small subset $Z_{n} \subseteq I_{n}$ such that $\partial\left(\left\langle Z_{n}\right\rangle\right) \supseteq \operatorname{Ker}\left(\left.\partial_{n-1}\right|_{\left\langle Z_{n-1}\right\rangle}\right)$.

The next step in the zigzag procedure consists of choosing a $\kappa$-small subset $Z_{n-1}^{(1)} \subseteq I_{n-1}$, containing $Z_{n-1}$, such that $\partial_{n}\left(\left\langle Z_{n}\right\rangle\right) \subseteq\left\langle Z_{n-1}^{(1)}\right\rangle$. Let $y \in \partial_{n}\left(\left\langle Z_{n}\right\rangle\right)$ of degree $b$. Then $y=\left(\partial_{n}\right)_{b}(z)$, where

$$
z=\sum_{i \in Z_{n}} r_{i} \cdot[i] .
$$

We have

$$
y=\sum_{i \in Z_{n}}\left(\partial_{n}\right)_{b}\left(r_{i} \cdot[i]\right) .
$$

On the other hand,

$$
\left(\partial_{n}\right)_{b}\left(r_{i} \cdot[i]\right)=\sum_{j \in Z_{n-1}^{(1), y, i}} q_{j} \cdot[j]
$$


for a finite set $Z_{n-1}^{(1), y, i} \subseteq I_{n-1}$. Thus,

$$
y=\sum_{i \in Z_{n}} \sum_{j \in Z_{n-1}^{(1), y, i}} q_{j} \cdot[j]=\sum_{j \in Z_{n-1}^{(1)}} q_{j} \cdot[j],
$$

where $Z_{n-1}^{(1)}$ is the disjoint union

$$
\bigsqcup\left\{Z_{n-1}^{(1), y, i}: y \in \partial_{n}\left(\left\langle Z_{n}\right\rangle\right) \text { and } i \in Z_{n}\right\} .
$$

We have

$$
\begin{aligned}
\operatorname{Card}\left(Z_{n-1}^{(1)}\right) & =\sum\left\{\operatorname{Card}\left(Z_{n-1}^{(1), y, i}\right): y \in \partial_{n}\left(\left\langle Z_{n}\right\rangle\right) \quad \text { and } i \in Z_{n}\right\} \\
& \leq \operatorname{Card}\left(\left\{\left(r_{i}: i \in Z_{n}\right): y \in \partial_{n}\left(\left\langle Z_{n}\right\rangle\right)\right\}\right) \leq \kappa .
\end{aligned}
$$

Then $Z_{n-1}^{(1)}$ is a $\kappa$-small subset of $I_{n-1}$ such that $\partial_{n}\left(\left\langle Z_{n}\right\rangle\right) \subseteq\left\langle Z_{n-1}^{(1)}\right\rangle$. Note that we can construct $Z_{n-1}^{(1)}$ containing $Z_{n-1}$. Similarly, there is a $\kappa$-small subset $Z_{n-2}^{(1)} \subseteq I_{n-2}$ containing $Z_{n-2}$ such that $\partial_{n-1}\left(\left\langle Z_{n-1}^{(1)}\right\rangle\right)$ $\subseteq\left\langle Z_{n-2}^{(1)}\right\rangle$.

At this point, we just need to mimic the argument given in the proof of [2, Proposition 4.1], with the corresponding considerations for $\operatorname{Mod}(\mathfrak{R})$. Set

$$
J_{k}:=Z_{k} \cup Z_{k}^{(1)} \cup \cdots
$$

for every $0 \leq k \leq n$. It is clear that $\left\langle J_{k}\right\rangle:=\bigoplus_{i \in J_{k}} \operatorname{Hom}_{\mathfrak{R}}([i],-)$ is a $\kappa$-small submodule of $\left\langle I_{k}\right\rangle$. By construction, we have an exact sequence

$$
0 \longrightarrow\left\langle J_{n}\right\rangle \stackrel{\partial_{n}}{\longrightarrow}\left\langle J_{n-1}\right\rangle \longrightarrow \cdots \longrightarrow\left\langle J_{1}\right\rangle \stackrel{\partial_{1}}{\longrightarrow}\left\langle J_{0}\right\rangle \stackrel{\partial_{0}}{\longrightarrow} N \longrightarrow 0,
$$

where $N=\operatorname{CoKer}\left(\left\langle J_{1}\right\rangle \stackrel{\partial_{1}}{\rightarrow}\left\langle J_{0}\right\rangle\right)$. Note that $x \in N$ and that each $\left\langle J_{k}\right\rangle$ is projective by Corollary 3.19. It remains to show that $M / N$ is $n$-projective. It suffices to take the quotient of the resolution of $M$ by the resolution of $N$, to get an exact sequence

$$
0 \longrightarrow \frac{\left\langle I_{n}\right\rangle}{\left\langle J_{n}\right\rangle} \longrightarrow \frac{\left\langle I_{n-1}\right\rangle}{\left\langle J_{n-1}\right\rangle} \longrightarrow \cdots \longrightarrow \frac{\left\langle I_{0}\right\rangle}{\left\langle J_{0}\right\rangle} \longrightarrow \frac{M}{N} \longrightarrow 0 .
$$

It is not hard to check that $\left\langle I_{k}\right\rangle /\left\langle J_{k}\right\rangle \cong\left\langle I_{k}-J_{k}\right\rangle$. So the previous sequence is a projective resolution of length $n$ of $M / N$. 
Proposition 3.25. Let $(\mathcal{A}, \mathcal{B})$ be a hereditary cotorsion pair of left modules over $\mathfrak{R}$ and $\kappa$ a regular cardinal satisfying the condition $\kappa>$ $\operatorname{Card}\left(\operatorname{Hom}_{\mathfrak{R}}(a, b)\right)$ for every $a, b \in \mathrm{Ob}(\mathfrak{R})$. Suppose, for each left $n$ $\mathcal{A}$-module $X$ and each $x \in X$, there exists a $\kappa$-small left $n$ - $\mathcal{A}$-module $X_{x} \subseteq X$ such that $x \in X_{x}$ and that $X / X_{x}$ is also a left $n$-A-module. Then every left $n-\mathcal{A}$-module is a transfinite extension of the class of $\kappa$-small left $n$-A-modules.

Proof. Let $X$ be a left $\mathfrak{R}$-module as described in the statement. Choose any $x_{0} \in X$. Then there exists a small left $n$ - $\mathcal{A}$-module $X_{0}$ such that $x_{0} \in X_{0}$ and such that $X / X_{0}$ is also a left $n$ - $\mathcal{A}$-module. Now choose a class $x_{1}+X_{0} \neq 0+X_{0}$. Then there exists a small left $n$-A-module $X_{1} / X_{0}$ such that $x_{1}+X_{0} \in X_{1} / X_{0}$ and such that $X / X_{1} \cong\left(X / X_{0}\right) /\left(X_{1} / X_{0}\right)$ is a left $n$ - $\mathcal{A}$-module. Note that $X_{0} \subseteq X_{1}$ and that $X_{1}$ is $\kappa$-small since $\operatorname{Card}\left(X_{1}\right)=\operatorname{Card}\left(X_{1} / X_{0}\right) \cdot \operatorname{Card}\left(X_{0}\right)$. Since we have a short exact sequence

$$
0 \longrightarrow X_{0} \longrightarrow X_{1} \longrightarrow X_{1} / X_{0} \longrightarrow 0,
$$

where $X_{0}$ and $X_{1} / X_{0}$ are left $n$ - $\mathcal{A}$-modules, by Lemma $2.3, X_{1}$ is also a left $n$-A-module. Using transfinite induction, we can construct a family of modules $\left(X_{\alpha}: \alpha<\lambda\right)$, for some ordinal $\lambda$, such that $X$ is the transfinite extension $X=\operatorname{CoLim}_{\alpha<\lambda} X_{\alpha}$.

The following theorem is proven in [2] for the category ${ }_{R}$ Mod. The arguments carry over easily to the category of modules over ringoids.

Theorem 3.26 ([2, Theorem 4.2] for modules over ringoids). The class of n-projective left $\mathfrak{R}$-modules $\mathcal{P}_{n}(\operatorname{Mod}(\mathfrak{R}))$ is the left half of a hereditary and complete cotorsion pair $\left(\mathcal{P}_{n}(\operatorname{Mod}(\mathfrak{R})),\left(\mathcal{P}_{n}(\operatorname{Mod}(\mathfrak{R}))\right)^{\perp}\right)$ cogenerated by a set of representatives of the class $\left(\mathcal{P}_{n}(\operatorname{Mod}(\mathfrak{R}))\right) \leq \kappa$.

4. n-projective model structures. The goal in this section is to construct a new Abelian model structure on $\mathbf{C h}\left({ }_{R} \mathbf{M o d}\right)$ where the weak equivalences are given by the quasi-isomorphisms and the trivial cofibrations by monomorphisms with $n$-projective cokernels. The motivation of this problem comes from the case $n=0$ studied by Hovey in [15, subsection 2.3]. 
Given two chain complexes $X$ and $Y$ in $\mathbf{C h}(\mathcal{C})$, the enriched homcomplex $\operatorname{Hom}^{\prime}(X, Y)$ is defined by

$$
\operatorname{Hom}^{\prime}(X, Y)_{m}:=\prod_{k \in \mathbb{Z}} \operatorname{Hom}_{\mathcal{C}}\left(X_{k}, Y_{m+k}\right)
$$

for every $m \in \mathbb{Z}$, with boundary maps

$$
\begin{aligned}
\partial_{m}^{\operatorname{Hom}^{\prime}(X, Y)}: \operatorname{Hom}^{\prime}(X, Y)_{m} & \longrightarrow \operatorname{Hom}^{\prime}(X, Y)_{m-1} \\
f=\left(f_{k}: X_{k}\right. & \left.\longrightarrow Y_{m+k}\right)_{k \in \mathbb{Z}} \\
& \longmapsto\left(\partial_{k+n}^{Y} \circ f_{k}-(-1)^{n} f_{k-1} \circ \partial_{k}^{X}\right)_{k \in \mathbb{Z} .}
\end{aligned}
$$

We recall the following notions given by Gillespie in [9, Definition 3.3]. Let $(\mathcal{A}, \mathcal{B})$ be a cotorsion pair in an Abelian category $\mathcal{C}$. A chain complex $X$ in $\mathbf{C h}(\mathcal{C})$ is:

(1) An $\mathcal{A}$-complex if $X$ is exact, and each cycle $Z_{m}(X)$ is in $\mathcal{A}$.

(2) A dg- $\mathcal{A}$-complex ("dg" for differential graded) if $X_{m} \in \mathcal{A}$ for each $m$ and every chain map $X \rightarrow B$ is homotopic to zero (or equivalently, the complex $\operatorname{Hom}^{\prime}(X, B)$ is exact) whenever $B$ is a $\mathcal{B}$-complex.

(3) A dg- $\mathcal{B}$-complex if $X_{m} \in \mathcal{B}$ for each $m$ and every chain map $A \rightarrow X$ is homotopic to zero (or equivalently, the complex $\operatorname{Hom}^{\prime}(A, X)$ is exact) whenever $A$ is an $\mathcal{A}$-complex.

Let $\widetilde{\mathcal{A}}, \operatorname{dg} \widetilde{\mathcal{A}}$ and $\operatorname{dg} \widetilde{\mathcal{B}}$ denote the classes of $\mathcal{A}$-complexes, dg- $\mathcal{A}$ complexes and dg- $\mathcal{B}$-complexes, respectively.

Example 4.1. By Proposition 3.6, $\widetilde{\mathcal{P}_{n}(\mathcal{C})}$ and $\widetilde{\mathcal{I}_{n}(\mathcal{C})}$ are the classes of $n$-projective and $n$-injective complexes in $\mathbf{C h}(\mathcal{C})$, respectively.

Consider the class $\mathcal{P}_{n}$ of $n$-projective left $R$-modules. We know by [2, Theorem 4.2] that $\left(\mathcal{P}_{n},\left(\mathcal{P}_{n}\right)^{\perp}\right)$ is a hereditary and complete cotorsion pair in ${ }_{R}$ Mod. So, by [9, Corollary 3.8 and Theorem 3.12], we have two compatible cotorsion pairs $\left(\widetilde{\mathcal{P}_{n}},\left(\widetilde{\mathcal{P}_{n}}\right)^{\perp}\right)$ and $\left(\operatorname{dg} \widetilde{\mathcal{P}_{n}},\left(\operatorname{dg} \widetilde{\mathcal{P}_{n}}\right)^{\perp}\right)$. By Example 3.9 (ii) and Proposition 3.26, we have the following result.

Corollary 4.2. The cotorsion pair $\left(\widetilde{\mathcal{P}_{n}},\left(\widetilde{\mathcal{P}_{n}}\right)^{\perp}\right)$ is hereditary and complete. 
The fact that the pair $\left(\operatorname{dg} \widetilde{\mathcal{P}_{n}},\left(\operatorname{dg} \widetilde{\mathcal{P}_{n}}\right)^{\perp}\right)$ is complete shall be a consequence of the following result.

Proposition 4.3. Let $(\mathcal{A} \cap \mathcal{W}, \mathcal{B})$ and $(\mathcal{A}, \mathcal{B} \cap \mathcal{W})$ be two compatible cotorsion pairs in an Ablian category $\mathcal{C}$.

(i) Suppose $\left({ }^{\perp} \mathcal{W}, \mathcal{W}\right)$ is a cotorsion pair cogenerated by a set $\mathcal{S}_{\mathcal{W}}$. If the pair $(\mathcal{A} \cap \mathcal{W}, \mathcal{B})$ is also cogenerated by a set $\mathcal{S}_{\mathcal{A} \cap \mathcal{W}}$, then $(\mathcal{A}, \mathcal{B} \cap \mathcal{W})$ is cogenerated by $\mathcal{S}_{\mathcal{A} \cap \mathcal{W}} \cup \mathcal{S}_{\mathcal{W}}$.

(ii) Suppose $\mathcal{C}$ has enough projective and injective objects. If both $\left({ }^{\perp} \mathcal{W}, \mathcal{W}\right)$ and $\left(\mathcal{W}, \mathcal{W}^{\perp}\right)$ are complete cotorsion pairs, then the pair $(\mathcal{A}, \mathcal{B} \cap \mathcal{W})$ is complete if, and only if, $(\mathcal{A} \cap \mathcal{W}, \mathcal{B})$ is.

Proof. Part (i) follows by

$$
\mathcal{B} \cap \mathcal{W}=\left(\mathcal{S}_{\mathcal{A} \cap \mathcal{W}}\right)^{\perp} \cap\left(\mathcal{S}_{\mathcal{W}}\right)^{\perp}=\left(\mathcal{S}_{\mathcal{A} \cap \mathcal{W}} \cup \mathcal{S}_{\mathcal{W}}\right)^{\perp}
$$

For part (ii), we only prove the implication $(\Rightarrow)$, since the other is dual. So suppose $(\mathcal{A}, \mathcal{B} \cap \mathcal{W})$ is complete, and let $X$ be an object in $\mathcal{C}$. Since $\left(\mathcal{W}, \mathcal{W}^{\perp}\right)$ is complete, there exists a short exact sequence

$$
0 \longrightarrow X \longrightarrow C \longrightarrow W \longrightarrow 0
$$

where $C \in \mathcal{W}^{\perp}$ and $W \in \mathcal{W}$. Since $(\mathcal{A}, \mathcal{B} \cap \mathcal{W})$ is complete, there exists a short exact sequence

$$
0 \longrightarrow B \longrightarrow A \longrightarrow W \longrightarrow 0
$$

where $A \in \mathcal{A}$ and $B \in \mathcal{B} \cap \mathcal{W}$. Taking the pullback of $C \rightarrow W$ and $A \rightarrow W$ and using [19, Proposition 2, page 203], we get two exact sequences

$$
\begin{aligned}
& 0 \longrightarrow X \longrightarrow C \times_{W} A \longrightarrow A \longrightarrow 0 \\
& 0 \longrightarrow B \longrightarrow C \times_{W} A \longrightarrow C \longrightarrow 0
\end{aligned}
$$

Since $\mathcal{W}$ is closed under extensions, we have $A \in \mathcal{A} \cap \mathcal{W}$. It suffices to show $C \times_{W} A \in \mathcal{B}$. Note that $\mathcal{A} \cap \mathcal{W} \subseteq \mathcal{W}$ implies $\mathcal{W}^{\perp} \subseteq(\mathcal{A} \cap \mathcal{W})^{\perp}=\mathcal{B}$. Then $C \in \mathcal{B}$. It follows that $C \times_{W} A \in \mathcal{B}$ since $\mathcal{B}$ is closed under extensions. Hence, $X \rightarrow C \times_{W} A$ is a special $\mathcal{B}$-pre-envelope. By [21, Corollary 2.4], $(\mathcal{A} \cap \mathcal{W}, \mathcal{B})$ is complete. 
Remark 4.4. A cotorsion pair $(\mathcal{A}, \mathcal{B})$ in an Abelian category $\mathcal{C}$ is functorially complete if, for every object $X$, there exist a special $\mathcal{A}$ pre-cover $A \rightarrow X$ and a special $\mathcal{B}$-pre-envelope $X \rightarrow B$ such that $A$ and $B$ are both functorial in $X$.

(i) Proposition 4.3 (ii) is also valid for functorially complete cotorsion pairs. In this sense, (ii) can also be stated as follows:

(ii') If $\mathcal{C}$ has functorially enough projective and injective objects, and if $\left({ }^{\perp} \mathcal{W}, \mathcal{W}\right)$ and $\left(\mathcal{W}, \mathcal{W}^{\perp}\right)$ are functorially complete, then $(\mathcal{A}, \mathcal{B} \cap \mathcal{W})$ is functorially complete if, and only if, $(\mathcal{A} \cap \mathcal{W}, \mathcal{B})$ is.

(ii) In the statement of Eklof and Trlifaj's theorem, cotorsion pairs cogenerated by sets are actually functorially complete.

(iii) To prove the "if" part of Hovey's correspondence, $(\mathcal{A} \cap \mathcal{W}, \mathcal{B})$ and $(\mathcal{A}, \mathcal{B} \cap \mathcal{W})$ need to be functorially complete (see $[\mathbf{1 4}$, Theorem $2.2])$.

To deduce that $\left(\operatorname{dg} \widetilde{\mathcal{P}_{n}},\left(\operatorname{dg} \widetilde{\mathcal{P}_{n}}\right)^{\perp}\right)$ is complete, consider the class $\mathcal{E}$ of exact chain complexes. The trivial cotorsion pairs $\left(\mathcal{P}_{0},{ }_{R} \mathbf{M o d}\right)$ and $\left({ }_{R} \operatorname{Mod}, \mathcal{I}_{0}\right)$ induce two pairs $\left(\operatorname{dg} \widetilde{\mathcal{P}_{0}},\left(\operatorname{dg} \widetilde{\mathcal{P}_{0}}\right)^{\perp}\right)$ and $\left({ }^{\perp}\left(\operatorname{dg} \widetilde{\mathcal{I}_{0}}\right), \operatorname{dg} \widetilde{\mathcal{I}_{0}}\right)$ by [9, Corollary 3.8]. In [8, Propositions 2.3.4, 2.3.5], it is proven that $\left(\operatorname{dg} \widetilde{\mathcal{P}_{0}}\right)^{\perp}=\mathcal{E}={ }^{\perp}\left(\operatorname{dg} \widetilde{\mathcal{I}_{0}}\right)$. On the one hand, $\left(\mathcal{E}, \operatorname{dg} \widetilde{\mathcal{I}_{0}}\right)$ is complete by $[\mathbf{8}$, Theorem 2.3.17]. On the other hand, in [4, Lemma 5.1], it is proven that $\left(\mathrm{dg} \widetilde{\mathcal{P}_{0}}, \mathcal{E}\right)$ is cogenerated by a set, and so complete. Then putting $\mathcal{W}=\mathcal{E}$ in the previous proposition, we have that $\left(\operatorname{dg} \widetilde{\mathcal{P}_{n}},\left(\operatorname{dg} \widetilde{\mathcal{P}_{n}}\right)^{\perp}\right)$ is a complete cotorsion pair (cogenerated by a set). Therefore, the following theorem follows by Hovey's correspondence.

Theorem 4.5. The two compatible and complete cotorsion pairs

$$
(\mathcal{A}, \mathcal{B} \cap \mathcal{W})=\left(\operatorname{dg} \widetilde{\mathcal{P}_{n}},\left(\operatorname{dg} \widetilde{\mathcal{P}_{n}}\right)^{\perp}\right)
$$

and

$$
(\mathcal{A} \cap \mathcal{W}, \mathcal{B})=\left(\widetilde{\mathcal{P}_{n}},\left(\widetilde{\mathcal{P}_{n}}\right)^{\perp}\right)
$$

give rise to a unique Abelian model category structure on $\mathbf{C h}\left({ }_{R} \mathbf{M o d}\right)$, called n-projective model structure, such that $\mathcal{A}=\operatorname{dg} \widetilde{\mathcal{P}_{n}}, \mathcal{B}=\left(\widetilde{\mathcal{P}_{n}}\right)^{\perp}$ 
and $\mathcal{W}=\mathcal{E}$ are classes of cofibrant, fibrant and trivial objects, respectively.

\section{Remark 4.6.}

(i) It is not hard to see that, in the previous model structure, weak equivalences are given by quasi-isomorphisms. Moreover, in every Abelian model category with $\mathcal{E}$ as the class of trivial objects, a map is a weak equivalence if, and only if, it is a quasiisomorphism. To see this, it is important to know that, in [14], weak equivalences in Abelian model structures are defined as the composition of trivial cofibrations followed by trivial fibrations.

(ii) So far we have only defined the fibrant objects of the previous model structure as those that have no extension from the trivially cofibrant objects. Using [9, Corollary 3.8], we have that an object $Y$ is fibrant if, and only if, each $Y_{m}$ has no extension from the $n$ projective modules and if every chain map $X \rightarrow Y$ is homotopic to 0 whenever $X$ is trivially cofibrant. In a similar way, we have that an object, $Y$, is trivially fibrant if, and only if, it is exact and if every cycle $Z_{m}(Y)$ has no extension from the $n$-projective modules.

An interesting question about a new model structure whether or not it is monoidal. In order to have a monoidal model structure on $\mathcal{C}$, we need $\mathcal{C}$ to be equipped with a symmetric monoidal structure. Roughly speaking, a symmetric monoidal structure on $\mathcal{C}$ is given by a tensor product $-\otimes-: \mathcal{C} \times \mathcal{C} \rightarrow \mathcal{C}$ and a unit object $S \in \mathrm{Ob}(\mathcal{C})$ such that, for every $X, Y, Z \in \mathrm{Ob}(\mathcal{C})$, we have isomorphisms

$$
\begin{aligned}
X \otimes(Y \otimes Z) & \cong(X \otimes Y) \otimes Z, \\
X \otimes Y & \cong Y \otimes X, \\
S \otimes X & \cong X,
\end{aligned}
$$

and

$$
X \otimes S \cong X,
$$

(see [15, Section 4.1] for details). The category ${ }_{R}$ Mod, with $R$ a commutative ring, is an example of a symmetric monoidal category. 
The tensor product is the standard tensor product $\otimes_{R}$ of modules, where $R$ is the unit.

We present two more examples of symmetric monoidal structures on $\mathbf{C h}\left({ }_{R}\right.$ Mod). Given two chain complexes $X \in \operatorname{Ob}\left(\mathbf{C h}\left(\boldsymbol{M o d}_{R}\right)\right)$ and $Y \in \mathrm{Ob}\left(\mathbf{C h}\left({ }_{R} \mathbf{M o d}\right)\right)$, the standard tensor product of $X$ and $Y$ is given by the chain complex $X \otimes Y$ in $\mathbf{C h}(\mathbf{A b})$ defined by

$$
(X \otimes Y)_{n}:=\bigoplus_{k \in \mathbb{Z}} X_{k} \otimes_{R} Y_{n-k},
$$

where the boundary maps are given by

$$
x \otimes y \longmapsto \partial_{k}^{X}(x) \otimes y+(-1)^{k} x \otimes \partial_{n-k}^{Y}(y),
$$

for every $x \otimes y \in X_{k} \otimes Y_{n-k}$. This construction defines a functor $-\otimes-$, from which one constructs the left derived functors $\operatorname{Tor}_{i}(-,-)$, with $i \geq 0$. From $\otimes$, the bar tensor product of $X$ and $Y$ is defined as the chain complex $X \bar{\otimes} Y$ in $\mathbf{C h}(\mathbf{A b})$ given by

$$
(X \bar{\otimes} Y)_{n}:=\frac{(X \otimes Y)_{n}}{B_{n}(X \otimes Y)},
$$

where the boundary maps are defined by

$$
x \otimes y+B_{n}(X \otimes Y) \longmapsto \partial_{k}^{X}(x) \otimes y+B_{n-1}(X \otimes Y),
$$

for every $x \otimes y+B_{n}(X \otimes Y) \in(X \bar{\otimes} Y)_{n}$. As far as the author knows, the definition of this tensor product first appeared in [8]. If $R$ is a commutative ring, then $\left(\mathbf{C h}\left({ }_{R} \mathbf{M o d}\right), \otimes\right)$ and $\left(\mathbf{C h}\left({ }_{R} \mathbf{M o d}\right), \bar{\otimes}\right)$ are symmetric monoidal categories, where $S^{0}(R)$ and $D^{1}(R)$ are the units with respect to $\otimes$ and $\bar{\otimes}$, respectively (see [8, Proposition 4.2.14] and [15, Proposition 4.2.13]).

A monoidal model category is a model category $\mathcal{C}$ equipped with a symmetric monoidal structure $(\otimes, S)$ such that the following conditions are satisfied:

(1) For each pair of maps $f: U \rightarrow V$ and $g: W \rightarrow X$, the pushout of $f \otimes \operatorname{id}_{W}$ and $\operatorname{id}_{U} \otimes g$ induces a map $f \square g$, making the following diagram commute: 


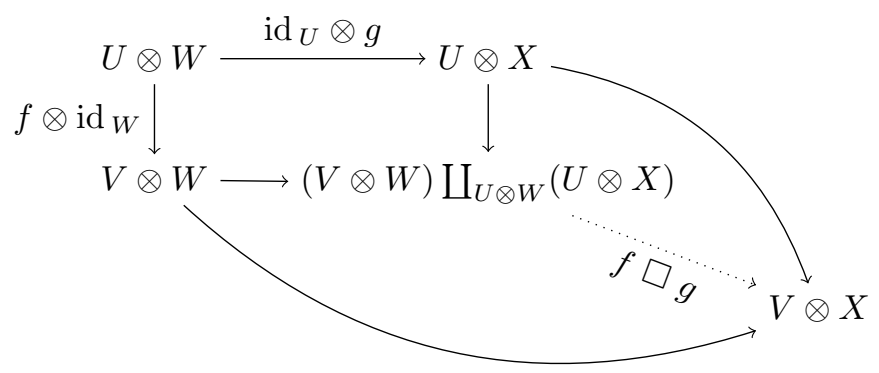

If, given cofibrations $f: U \rightarrow V$ and $g: W \rightarrow X$ in $\mathcal{C}$, the induced map $f \square g$ is a cofibration, which is trivial if either $f$ or $g$ is.

(2) Using functorial factorizations, write

$$
0 \longrightarrow S=0 \longrightarrow Q(S) \stackrel{q}{\longrightarrow} S
$$

as the composition of a cofibration followed by a trivial fibration. Then the maps $q \otimes X: Q(S) \otimes X \rightarrow S \otimes X$ and $X \otimes q: X \otimes Q(S) \rightarrow X \otimes S$ are weak equivalences for all cofibrant objects $X$.

It is known that the $n$-projective model structure is monoidal with respect to the usual tensor product on $\mathbf{C h}\left({ }_{R} \mathbf{M o d}\right)$ if $n=0$ (see [15, Chapter 4]). In general, this is not the case if $n>0$. For instance, consider $n=1$ and $R=\mathbb{Z}$. Note that $\mathbb{Z}_{2}$ is a 1 -projective $\mathbb{Z}$-module, since it is not projective, and there exists a short exact sequence

$$
0 \longrightarrow \mathbb{Z} \stackrel{2 \times}{\longrightarrow} \mathbb{Z} \stackrel{\pi}{\longrightarrow} \mathbb{Z}_{2} \longrightarrow 0
$$

where $2 \times$ is the map $x \mapsto 2 \times x$ and $\pi$ is the canonical projection $x \mapsto \bar{x} \in\{0,1\}$. Now let $X$ be the complex given by the previous sequence, where $X_{1}=\mathbb{Z}, X_{0}=\mathbb{Z}$ and $X_{-1}=\mathbb{Z}_{2}$. We have $X \in$ $\widetilde{\mathcal{P}_{1}}$. Consider also the complex $S^{0}\left(\mathbb{Z}_{2}\right)$. Then $S^{0}\left(\mathbb{Z}_{2}\right) \in \operatorname{dg} \widetilde{\mathcal{P}_{1}}$ since $\operatorname{Ext}_{\mathbf{C h}\left(\operatorname{Mod}_{\mathbb{Z}}\right)}^{1}\left(S^{0}\left(\mathbb{Z}_{2}\right), Y\right) \cong \operatorname{Ext}_{\mathbb{Z}}^{1}\left(\mathbb{Z}_{2}, Z_{0} Y\right)$ (by [10, Lemma 4.2]) and $\operatorname{Ext}_{\mathbb{Z}}^{1}\left(\mathbb{Z}_{2}, Z_{0} Y\right)=0$ for every $Y \in \widetilde{\mathcal{P}_{1}^{\perp}}=\left(\operatorname{dg} \widetilde{\mathcal{P}_{1}}\right)^{\perp}$. It is not hard to see that

$$
\left(S^{0}\left(\mathbb{Z}_{2}\right) \otimes X\right)_{m}= \begin{cases}\mathbb{Z}_{2} \otimes_{\mathbb{Z}} \mathbb{Z} & \text { if } m=0 \text { or } 1 \\ \mathbb{Z}_{2} \otimes_{\mathbb{Z}} \mathbb{Z}_{2} & \text { if } m=-1 \\ 0 & \text { otherwise }\end{cases}
$$


and that $\partial_{1}^{S^{0}\left(\mathbb{Z}_{2}\right) \otimes X}$ is the zero map, so the sequence

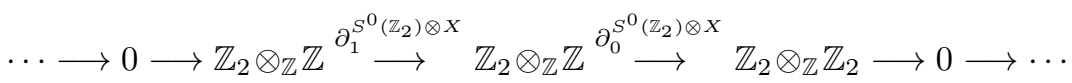

is not exact. We have a cofibration $0 \rightarrow S^{0}\left(\mathbb{Z}_{2}\right)$ and a trivial cofibration $0 \rightarrow X$, but the induced map

$$
\left(0 \longrightarrow S^{0}\left(\mathbb{Z}_{2}\right)\right) \square(0 \longrightarrow X)=0 \longrightarrow S^{0}\left(\mathbb{Z}_{2}\right) \otimes X
$$

is a cofibration not a weak equivalence, since $S^{0}\left(\mathbb{Z}_{2}\right) \otimes X$ is not exact. Therefore, the $n$-projective model structure on $\mathbf{C h}\left(\mathbf{M o d}_{\mathbb{Z}}\right)$ is not monoidal with respect to the tensor product $\otimes$. We can conclude the same for $\mathbf{C h}\left(\mathbf{M o d}_{\mathbb{Z}}\right)$ with respect to the bar tensor product $\bar{\otimes}$, since

$$
\left(S^{0}\left(\mathbb{Z}_{2}\right) \bar{\otimes} X\right)_{m}= \begin{cases}\mathbb{Z}_{2} \otimes_{\mathbb{Z}} \mathbb{Z} & \text { if } m=0 \text { or } 1, \\ 0 & \text { otherwise }\end{cases}
$$

and

$$
S^{0}\left(\mathbb{Z}_{2}\right) \bar{\otimes} X=\cdots \longrightarrow 0 \longrightarrow \mathbb{Z}_{2} \otimes_{\mathbb{Z}} \mathbb{Z}^{\partial_{1}^{S^{0}\left(\mathbb{Z}_{2}\right) \bar{\otimes} X}} \longrightarrow \mathbb{Z}_{2} \otimes_{\mathbb{Z}} \mathbb{Z} \longrightarrow 0 \longrightarrow \cdots,
$$

where $\partial_{1}^{S^{0}\left(\mathbb{Z}_{2}\right) \bar{\otimes} X}$ is the zero map. Hence, $S^{0}\left(\mathbb{Z}_{2}\right) \bar{\otimes} X$ is not an exact complex, and so the induced chain map

$$
0 \longrightarrow S^{0}\left(\mathbb{Z}_{2}\right) \bar{\otimes} X=\left(0 \longrightarrow S^{0}\left(\mathbb{Z}_{2}\right)\right) \square(0 \longrightarrow X)
$$

is not a trivial cofibration.

It is time to give an application of the $n$-projective model structure in the context of homotopy theory. Consider the category $\mathbf{C h}\left({ }_{R} \mathbf{M o d}\right)$ equipped with this model structure. This setting provides another way to compute the extension groups $\operatorname{Ext}_{R}^{i}(M, N)$ for every pair of left $R$-modules $M$ and $N$, in which we resolve $M$ by the class of $n$-projective modules. Recall that $\operatorname{Ext}_{R}^{i}(M, N)$ is given by the $i$ th cohomology group of the complex $\operatorname{Hom}_{R}\left(P_{\bullet}, N\right)$, or equivalently, by $\operatorname{Ext}^{i}(M, N)=H_{-i}\left(\operatorname{Hom}_{R}\left(P_{\bullet}, N\right)\right)$, where $P_{\bullet}$ is the chain complex obtained from a left projective resolution of $M$ after deleting the term $M$. On the other hand, it is not hard to see that $H_{-i}\left(\operatorname{Hom}_{R}\left(P_{\bullet}, N\right)\right)$ is isomorphic to the quotient $\operatorname{Hom}_{\mathbf{C h}}\left(_{R}\right.$ Mod $)\left(P_{\bullet}, S^{i}(N)\right) / \sim$, where $\sim$ is the equivalence relation given by the chain homotopy equivalence. We have $\operatorname{Ext}_{R}^{i}(M, N) \cong \operatorname{Hom}_{\mathbf{C h}\left({ }_{R} \text { Mod }\right)}\left(P \bullet, S^{i}(N)\right) / \sim$, where the 
right side term can be described by using the language of homotopy categories.

Let Ho $\left(\mathbf{C h}\left({ }_{R} \mathbf{M o d}\right)\right)$ denote the homotopy category associated to the model category $\mathbf{C h}\left({ }_{R} \mathbf{M o d}\right)$. We assume the reader is familiar with the notion of homotopy categories, although a good introduction to this field is given in [5]. To compute $\operatorname{Ext}_{R}^{i}(M, N)$ by resolving $M$ by $n$-projective modules, we first point out that there exists a bijection

$$
\mathbf{H o}\left(\mathbf{C h}\left({ }_{R} \mathbf{M o d}\right)\right)\left(S^{0}(M), S^{i}(N)\right) \cong \operatorname{Hom}_{\mathbf{C h}\left({ }_{R} \mathbf{M o d}\right)}(\mathbf{Q}, \mathbf{R}) / \sim,
$$

where $\mathbf{Q}$ is a cofibrant replacement of $S^{0}(M)$ and $\mathbf{R}$ a fibrant replacement of $S^{i}(N)$. We can have a left $n$-projective resolution

$$
\cdots \longrightarrow L_{1} \longrightarrow L_{0} \longrightarrow M \longrightarrow 0
$$

of $M$ with each cycle module in $\left(\mathcal{P}_{n}\right)^{\perp}$ and a right $\left(\mathcal{P}_{n}\right)^{\perp}$-resolution

$$
0 \longrightarrow N \longrightarrow J^{0} \longrightarrow J^{1} \longrightarrow \cdots
$$

of $N$ with each cycle module in $\mathcal{P}_{n}$, since $\left(\mathcal{P}_{n},\left(\mathcal{P}_{n}\right)^{\perp}\right)$ is a complete cotorsion pair. Deleting $M$ and $N$ in the previous complexes and using [9, Lemma 5.3], we have that

$$
\mathbf{L}=\cdots \longrightarrow Q_{1} \longrightarrow Q_{0} \longrightarrow 0
$$

is a cofibrant replacement of $S^{0}(M)$ and that the $i$ th suspension $\Sigma^{i}(\mathbf{J})$ of

$$
\mathbf{J}=0 \longrightarrow R^{0} \longrightarrow R^{1} \longrightarrow \cdots
$$

is a fibrant replacement of $S^{i}(N)$. In this sense, we obtain

$$
\operatorname{Ho}\left(\mathbf{C h}\left({ }_{R} \mathbf{M o d}\right)\right)\left(S^{0}(M), S^{i}(N)\right) \cong \operatorname{Hom}_{\mathbf{C h}\left({ }_{R} \mathbf{M o d}\right)}\left(\mathbf{L}, \Sigma^{i}(\mathbf{J})\right) / \sim .
$$

Recall that the $i$ th suspension of a chain complex $X$ is defined as the chain complex $\Sigma^{i}(X)$ where $\left(\Sigma^{i}(X)\right)_{m}=X_{m-i}$ and where the boundary maps are given by $\partial_{m}^{\Sigma^{i}(X)}:=(-1)^{i} \partial_{m-i}^{X}$, for every $m \in \mathbb{Z}$.

Using the arguments given by Gillespie in [9, Section 5], we have

$$
\operatorname{Ext}_{R}^{i}(M, N) \cong \operatorname{Hom}_{\mathbf{C h}\left({ }_{R} \mathbf{M o d}\right)}\left(\mathbf{L}, \Sigma^{i}(\mathbf{J})\right) / \sim .
$$

Finally, by [9, Lemma 2.1], we obtain the isomorphism $\operatorname{Ext}_{R}^{i}(M, N) \cong$ $H_{-i}\left(\operatorname{Hom}^{\prime}(\mathbf{L}, \mathbf{J})\right)$. The only inconvenience with this expression is that the terms of $\mathbf{J}$ are not described as easily as those of $\mathbf{L}$. 
We conclude this section by presenting the dual of the $n$-projective model structure. The case of the injective dimension is easier to study. Assume for the rest of this section that $\mathcal{C}$ is a Grothendieck category with a generator $G$. Grothendieck categories have enough injective objects. This was proven by Grothendieck in his famous paper (see [12, Theorem 1.10.1]). Moreover, in that paper, the validity of the Baer criterion is proven for any Grothendieck category (see [12, Lemma 1, page 136]). The next result follows.

Proposition 4.7. Let $\mathcal{C}$ be a Grothendieck category with a generator $G$. Then an object $Y$ of $\mathcal{C}$ is $n$-injective if, and only if, $\operatorname{Ext}_{\mathcal{C}}^{n+1}(G / J, Y)=0$ for every sub-object $J$ of $G$.

Corollary 4.8. If $\mathcal{C}$ is a Grothendieck category with generator $G$, then $\mathcal{I}_{n}(\mathcal{C})$ is the right half of a cotorsion pair $\left({ }^{\perp}\left(\mathcal{I}_{n}(\mathcal{C})\right), \mathcal{I}_{n}(\mathcal{C})\right)$ cogenerated by a set of representatives of the class of all $S \in \Omega^{n}(G / J)$ with $J$ running over the set of all sub-objects of $G$.

It is not hard to see that the class $\mathcal{I}_{n}(\mathcal{C})$ is coresolving, and hence the pair $\left({ }^{\perp}\left(\mathcal{I}_{n}(\mathcal{C})\right), \mathcal{I}_{n}(\mathcal{C})\right)$ is also hereditary. If, in addition, $\mathcal{C}$ has enough projective objects, [9, Corollary 3.8] and Proposition 4.8 imply that $\left.\left(\mathrm{dg} \perp \widehat{\left(\mathcal{I}_{n}(\mathcal{C})\right.}\right), \widehat{\mathcal{I}_{n}(\mathcal{C})}\right)$ and $\left.\left(\perp \widehat{\left(\mathcal{I}_{n}(\mathcal{C})\right.}\right), \mathrm{dg} \widehat{\mathcal{I}_{n}(\mathcal{C})}\right)$ are compatible cotorsion pairs in $\mathbf{C h}(\mathcal{C})$. Moreover, by Proposition 3.6, we know $\widetilde{\mathcal{I}_{n}(\mathcal{C})}=$ $\mathcal{I}_{n}(\mathbf{C h}(\mathcal{C}))$. It follows by Corollary 4.8 , the pair $\left.\left(\operatorname{dg} \perp \widetilde{\left(\mathcal{I}_{n}(\mathcal{C})\right.}\right), \widetilde{\mathcal{I}_{n}(\mathcal{C})}\right)$ is complete. Hence, $\left(\widetilde{\perp\left(\mathcal{I}_{n}\right)}, \operatorname{dg} \widetilde{\mathcal{I}_{n}}\right)$ is a complete cotorsion pair by Proposition 4.3. Then Theorem 4.9 follows.

Theorem 4.9. The two compatible and complete cotorsion pairs,

$$
(\mathcal{A}, \mathcal{B} \cap \mathcal{W})=\left({ }^{\perp}\left(\widetilde{\mathcal{I}_{n}}\right), \widetilde{\mathcal{I}_{n}}\right)
$$

and

$$
(\mathcal{A} \cap \mathcal{W}, \mathcal{B})=\left({ }^{\perp}\left(\operatorname{dg} \widetilde{\mathcal{I}_{n}}\right), \operatorname{dg} \widetilde{\mathcal{I}_{n}}\right),
$$

give rise to a unique Abelian model category structure on $\mathbf{C h}\left({ }_{R} \mathbf{M o d}\right)$, called $n$-injective model structure, such that $\mathcal{A}={ }^{\perp}\left(\widetilde{\mathcal{I}_{n}}\right), \mathcal{B}=\operatorname{dg} \widetilde{\mathcal{I}_{n}}$ 
and $\mathcal{W}=\mathcal{E}$ are the classes of cofibrant, fibrant and trivial objects, respectively.

Using [9, Corollary 3.8], we have that an object $X$ is cofibrant if, and only if, each $X_{m}$ has no extension from the $n$-injective modules and if every chain map $X \rightarrow Y$ is homotopic to 0 whenever $Y$ is trivially fibrant. In a similar way, we have that an object $X$ is trivially cofibrant if, and only if, it is exact and if every cycle $Z_{m}(X)$ has no extension from the $n$-injective modules.

As with the $n$-projective model structure, we can present another way to compute $\operatorname{Ext}_{R}^{i}(M, N)$ by using fibrant and cofibrant replacements in the $n$-injective model structure. Specifically, we have

$$
\operatorname{Ext}_{R}^{i}(M, N) \cong H_{-i}\left(\operatorname{Hom}^{\prime}(\mathbf{T}, \mathbf{D})\right),
$$

where $\mathbf{D}$ is obtained by deleting $N$ in a right $n$-injective resolution of $N$ with cycles in ${ }^{\perp}\left(\mathcal{I}_{n}\right)$, and $\mathbf{T}$ by deleting $M$ in a left ${ }^{\perp}\left(\mathcal{I}_{n}\right)$-resolution of $M$ with cycles in $\mathcal{I}_{n}$.

5. Degreewise $n$-projective model structures. This section is devoted to constructing an Abelian model structure on $\mathbf{C h}\left({ }_{R} \mathbf{M o d}\right)$ where the exact complexes are trivial objects, and the cofibrant objects are chain complexes whose terms have projective dimension at most $n$. This structure represents an extension of the model structure found by Enochs, et al., in [4, Theorem 5.5]. For this purpose, it is useful to recall more induced cotorsion pairs in $\mathbf{C h}(\mathcal{C})$ from a cotorsion pair $(\mathcal{A}, \mathcal{B})$ in $\mathcal{C}$, along with some of their properties.

Let $\mathcal{A}$ be a class of objects in an Abelian category $\mathcal{C}$. Let $\operatorname{dw} \widetilde{\mathcal{A}}$ denote the class of all complexes $X$ in $\mathbf{C h}(\mathcal{C})$ such that $X_{m} \in \mathcal{A}$ for every $m \in \mathbb{Z}$. We shall say that a complex in $\mathrm{dw} \widetilde{\mathcal{A}}$ is a degreewise $\mathcal{A}$-complex, or a dw-A-complex. The class ex $\widetilde{\mathcal{A}}:=\mathrm{dw} \widetilde{\mathcal{A}} \cap \mathcal{E}$ shall be called the class of exact $\mathrm{dw}-\mathcal{A}$-complexes. These definitions were given by Gillespie in [10, Definition 3.1].

In [10, Proposition 3.2], it is proven by Gillespie that, if $(\mathcal{A}, \mathcal{B})$ is a cotorsion pair in an Abelian category $\mathcal{C}$, then $\left(\operatorname{dw} \widetilde{\mathcal{A}},(\operatorname{dw} \widetilde{\mathcal{A}})^{\perp}\right)$ and $\left({ }^{\perp}(\mathrm{dw} \widetilde{\mathcal{B}}), \mathrm{dw} \widetilde{\mathcal{B}}\right)$ are cotorsion pairs in $\mathbf{C h}(\mathcal{C})$. The same author proved in [10, Proposition 3.3] that if, in addition, $\mathcal{B}$ contains a cogenerator of finite injective dimension, then $\left(\operatorname{ex} \widetilde{\mathcal{A}},(\text { ex } \widetilde{\mathcal{A}})^{\perp}\right)$ is a cotorsion pair. This 
is also true if we assume instead that $\mathcal{C}$ has enough injective objects. Dually, if either $\mathcal{A}$ contains a generator of finite projective dimension or $\mathcal{C}$ has enough projective objects, then $\left({ }^{\perp}(\operatorname{ex} \widetilde{\mathcal{B}})\right.$, ex $\left.\widetilde{\mathcal{B}}\right)$ is a cotorsion pair in $\mathbf{C h}(\mathcal{C})$.

Considering the trivial cotorsion pair $\left(\mathcal{P}_{0},{ }_{R}\right.$ Mod $)$ in ${ }_{R}$ Mod, we have two cotorsion pairs $\left(\mathrm{dw} \widetilde{\mathcal{P}_{0}},\left(\mathrm{dw} \widetilde{\mathcal{P}_{0}}\right)^{\perp}\right)$ and $\left(\right.$ ex $\left.\widetilde{\mathcal{P}_{0}},\left(\text { ex } \widetilde{\mathcal{P}_{0}}\right)^{\perp}\right)$ in the category $\mathbf{C h}\left({ }_{R} \mathbf{M o d}\right)$. In [4], the authors show that these two pairs are cogenerated by sets. Concerning the former pair, they prove in $[4$, Theorem 4.4] that if $P \in \mathrm{dw} \widetilde{\mathcal{P}_{0}}$ and if $\mathcal{S}$ is a set of representatives of complexes with all terms countably generated projective modules, then $P$ has an $\mathcal{S}$-filtration. For the latter pair, if $\kappa$ is an infinite cardinal with $\kappa>\operatorname{Card}(R)$, then every $P \in$ ex $\widetilde{\mathcal{P}_{0}}$ has a $\left(\right.$ ex $\left.\widetilde{\mathcal{P}_{0}}\right) \leq \kappa$-filtration (see [4, Theorem 4.6]). It follows by Proposition 2.5 that $\left(\mathrm{dw} \widetilde{\mathcal{P}_{0}},\left(\mathrm{dw} \widetilde{\mathcal{P}_{0}}\right)^{\perp}\right)$ and $\left(\right.$ ex $\left.\widetilde{\mathcal{P}_{0}},\left(\text { ex } \widetilde{\mathcal{P}_{0}}\right)^{\perp}\right)$ are complete cotorsion pairs. In [4] it is also proven that these pairs are compatible. So it follows by Hovey's correspondence the existence of a model structure on $\mathbf{C h}\left({ }_{R} \mathbf{M o d}\right)$, which shall be referred to as the degreewise projective model structure, where the (trivial) cofibrations are the monomorphisms with cokernels in $\mathrm{dw} \widetilde{\mathcal{P}_{0}}$ (in ex $\widetilde{\mathcal{P}_{0}}$ ), the (trivial) fibrations are the epimorphisms with kernels in $\left(\text { ex } \widetilde{\mathcal{P}_{0}}\right)^{\perp}\left(\right.$ in $\left.\left(\mathrm{dw} \widetilde{\mathcal{P}_{0}}\right)^{\perp}\right)$, and the weak equivalences are quasiisomorphisms (see [4, Theorem 5.5]).

We generalize the arguments given in [4] to any projective dimension. In other words, we shall prove that every (exact) degreewise $n$-projective complex is filtered by some set and then shall conclude that the two cotorsion pairs

$$
\left(\operatorname{ex} \widetilde{\mathcal{P}_{n}},\left(\operatorname{ex} \widetilde{\mathcal{P}_{n}}\right)^{\perp}\right) \quad \text { and } \quad\left(\mathrm{dw} \widetilde{\mathcal{P}_{n}},\left(\mathrm{dw} \widetilde{\mathcal{P}_{n}}\right)^{\perp}\right)
$$

are compatible and complete, in order to obtain the following result:

Theorem 5.1. The two compatible and complete cotorsion pairs

$$
(\mathcal{A}, \mathcal{B} \cap \mathcal{W})=\left(\mathrm{dw} \widetilde{\mathcal{P}_{n}},\left(\mathrm{dw} \widetilde{\mathcal{P}_{n}}\right)^{\perp}\right)
$$

and

$$
(\mathcal{A} \cap \mathcal{W}, \mathcal{B})=\left(\operatorname{ex} \widetilde{\mathcal{P}_{n}},\left(\operatorname{ex} \widetilde{\mathcal{P}_{n}}\right)^{\perp}\right)
$$


give rise to a unique Abelian model category structure on $\mathbf{C h}\left({ }_{R} \mathbf{M o d}\right)$, called degreewise n-projective model structure, such that $\mathcal{A}=\mathrm{dw} \widetilde{\mathcal{P}_{n}}$, $\mathcal{B}=\left(\operatorname{ex} \widetilde{\mathcal{P}_{n}}\right)^{\perp}$ and $\mathcal{W}=\mathcal{E}$ are the classes of cofibrant, fibrant and trivial objects, respectively.

We first need to note that, by [10, Proposition 3.2], we have two cotorsion pairs $\left(\mathrm{dw} \widetilde{\mathcal{P}_{n}},\left(\mathrm{dw} \widetilde{\mathcal{P}_{n}}\right)^{\perp}\right)$ and $\left(\operatorname{ex} \widetilde{\mathcal{P}_{n}},\left(\operatorname{ex} \widetilde{\mathcal{P}_{n}}\right)^{\perp}\right)$.

Definition 5.2. We say that a chain complex $X$ in $\mathbf{C h}\left({ }_{R} \mathbf{M o d}\right)$ is degreewise $n$-projective if $X \in \mathrm{dw} \widetilde{\mathcal{P}_{n}}$.

The proof of [4, Theorem 4.4] is based on the following result by Kaplansky on projective modules. Recall that a left $R$-module is said to be countably generated if it is generated as a module by a countable subset.

Theorem 5.3 (see [18]). If $P$ is a projective module, then $P$ is the direct sum of countably generated projective modules.

So, when one thinks of a possible generalization of the degreewise projective model structure for $n$-projective modules, a good question would be whether it is possible to generalize Kaplansky's theorem for such modules.

Let $M \in \mathcal{P}_{n}$ be an $n$-projective module, so we can choose a projective resolution of length $n$, say

$$
0 \longrightarrow P_{n} \longrightarrow \cdots \longrightarrow P_{1} \longrightarrow P_{0} \longrightarrow M \longrightarrow 0 .
$$

By Kaplansky's theorem, we can write

$$
P_{k}=\bigoplus_{i \in I_{k}} P_{k}^{i}
$$

where $P_{k}^{i}$ is a countably generated projective module, for every $i \in I_{k}$ and every $0 \leq k \leq n$. Then we can rewrite the previous resolution as

$$
0 \longrightarrow \bigoplus_{i \in I_{n}} P_{n}^{i} \longrightarrow \cdots \longrightarrow \bigoplus_{i \in I_{1}} P_{1}^{i} \longrightarrow \bigoplus_{i \in I_{0}} P_{0}^{i} \longrightarrow M \longrightarrow 0 .
$$


From now on, we shall write any projective resolution of length $n$ by using such direct sum decompositions. We shall denote by $\mathcal{P}_{n}^{\aleph_{0}}$ the class of all modules, $M$, having a projective resolution as above, where $I_{k}$ is a countable set for each $0 \leq k \leq n$.

We shall prove that $\left(\mathrm{dw} \widetilde{\mathcal{P}_{n}},\left(\mathrm{dw} \widetilde{\mathcal{P}_{n}}\right)^{\perp}\right)$ is a cotorsion pair cogenerated by a set of representatives of the class $\mathrm{dw} \widetilde{\mathcal{P}_{n}^{\aleph_{0}}}$, by showing that every complex in $\mathrm{dw} \widetilde{\mathcal{P}_{n}}$ is $\mathrm{dw} \widetilde{\mathcal{P}_{n}^{\aleph_{0}}}$-filtered. We need the following extension of Kaplansky's theorem for $n$-projective modules.

Proposition 5.4 (Kaplansky's theorem for $n$-projective modules). Let $R$ be a Noetherian ring. Let $M \in \mathcal{P}_{n}$, and let $N \subseteq M$ be a countably generated submodule of $M$. Then there exists a $\overline{\mathcal{P}}_{n}^{\aleph_{0}}$-filtration of $M$, $\left(M_{\alpha}: \alpha<\lambda\right)$ with $\lambda>1$, such that $M_{1} \in \mathcal{P}_{n}^{\aleph_{0}}$ and $N \subseteq M_{1}$.

Proof. Let $M \in \mathcal{P}_{n}$ exist with a finite projective resolution where each projective term $P_{k}$ is written as a direct sum $\bigoplus_{i \in I_{k}} P_{k}^{i}$ of countably generated projective modules, for every $0 \leq i \leq n$. We shall construct a $\mathcal{P}_{n}^{\aleph_{0}}$-filtration $\left(M_{\alpha}: \alpha<\lambda\right)$ of $M$, with $N \subseteq M_{1}$, by using the zigzag procedure described in the proof of Lemma 3.24.

Set $M_{0}=0$. To construct $M_{1}$, consider a countable set $\mathcal{G}$ of generators of $N$. Since $f_{0}$ is surjective, for every $g \in \mathcal{G}$, we can choose

$$
y_{g} \in \bigoplus_{i \in I_{0}} P_{0}^{i}
$$

such that $g=f_{0}\left(y_{g}\right)$. Consider the set

$$
Y=\left\{y_{g}: g \in \mathcal{G}\right\} .
$$

Since $Y$ is a countable subset of

$$
\bigoplus_{i \in I_{0}} P_{0}^{i},
$$

we have that $\langle Y\rangle$ is a countably generated submodule of $P_{0}$. Choose a countable subset $I_{0}^{1,0} \subseteq I_{0}$ such that

$$
\langle Y\rangle \subseteq \bigoplus_{i \in I_{0}^{1,0}} P_{0}^{i} .
$$


Then $f_{0}(\langle Y\rangle) \subseteq N$. Consider

$$
\operatorname{Ker}\left(\left.f_{0}\right|_{i \in I_{0}^{1,0}} P_{0}^{i}\right) \text {. }
$$

Since $\bigoplus_{i \in I_{0}^{1,0}} P_{0}^{i}$ is countably generated and $\operatorname{Ker}\left(\left.f_{0}\right|_{\bigoplus_{i \in I_{0}^{1,0}} P_{0}^{i}}\right)$ is a submodule of $\bigoplus_{i \in I_{0}^{1,0}} P_{0}^{i}$, we have that the kernel $\operatorname{Ker}\left(\left.f_{0}\right|_{\bigoplus_{i \in I_{0}^{1,0}} P_{0}^{i}}\right)$ is also countably generated, since $R$ is Noetherian.

Let $\mathcal{B}$ be a countable set of generators of $\operatorname{Ker}\left(\left.f_{0}\right|_{\bigoplus_{i \in I_{0}^{1,0}} P_{0}^{i}}\right)$. Let $b \in \mathcal{B}$. Then $f(b)=0$ and, by exactness of the above sequence, there exists

$$
y_{b} \in \bigoplus_{i \in I_{1}} P_{1}^{i}
$$

such that $b=f_{1}\left(y_{b}\right)$. Let $Y^{\prime}=\left\{y_{b}: b \in \mathcal{B}\right\}$. Note that $Y^{\prime}$ is a countable subset of

$$
\left(f_{1}\right)^{-1}\left(\operatorname{Ker}\left(\left.f_{0}\right|_{i \in I_{0}^{1,0}} P_{0}^{i}\right)\right)
$$

Then $\left\langle Y^{\prime}\right\rangle$ is a countably generated submodule of $\bigoplus_{i \in I_{1}} P_{1}^{i}$. Hence, there exists a countable subset $I_{1}^{1,0} \subseteq I_{1}$ such that

$$
\bigoplus_{i \in I_{1}^{1,0}} P_{1}^{i} \supseteq\left\langle Y^{\prime}\right\rangle
$$

Thus,

$$
f_{1}\left(\bigoplus_{i \in I_{1}^{1,0}} P_{1}^{i}\right) \supseteq f_{1}\left(\left\langle Y^{\prime}\right\rangle\right) \supseteq \operatorname{Ker}\left(\left.f_{0}\right|_{\bigoplus_{i \in I_{0}^{1,0}} P_{0}^{i}}\right)
$$

Repeat the same argument until a countable subset $I_{n}^{1,0} \subseteq I_{n}$ can be found such that

$$
f_{n}\left(\bigoplus_{i \in I_{n}^{1,0}} P_{n}^{i}\right) \supseteq \operatorname{Ker}\left(\left.f_{n-1}\right|_{\bigoplus_{i \in I_{n-1}^{1,0}} P_{n-1}^{i}}\right) .
$$

Now,

$$
f_{n}\left(\bigoplus_{i \in I_{n}^{1,0}} P_{n}^{i}\right)
$$


is a countably generated submodule of

$$
\bigoplus_{i \in I_{n-1}} P_{n-1}^{i}
$$

Then choose a countable subset $I_{n-1}^{1,0} \subseteq I_{n-1}^{1,1} \subseteq I_{n-1}$ with

$$
f_{n}\left(\bigoplus_{i \in I_{n}^{1,0}} P_{n}^{i}\right) \subseteq \bigoplus_{i \in I_{n-1}^{1,1}} P_{n-1}^{i}
$$

Continue this zigzag procedure infinitely many times, and set

$$
I_{k}^{1}=\bigcup_{m \geq 0} I_{k}^{1, m}
$$

for every $0 \leq k \leq n$. By construction, we get an exact sequence,

$$
0 \longrightarrow \bigoplus_{i \in I_{n}^{1}} P_{n}^{i} \longrightarrow \cdots \longrightarrow \bigoplus_{i \in I_{1}^{1}} P_{1}^{i} \longrightarrow \bigoplus_{i \in I_{0}^{1}} P_{0}^{i} \longrightarrow M_{1} \longrightarrow 0
$$

where

$$
x \in M_{1}:=\operatorname{CoKer}\left(\bigoplus_{i \in I_{1}^{1}} \longrightarrow \bigoplus_{i \in I_{0}^{1}} P_{0}^{i}\right) \subseteq M
$$

and $N \subseteq M_{1}$. We take the quotient of the resolution of $M$ by the resolution of $M^{\prime}$ and get a sequence

$$
0 \longrightarrow \bigoplus_{i \in I_{n}-I_{n}^{1}} P_{n}^{i} \longrightarrow \cdots \longrightarrow \bigoplus_{i \in I_{1}-I_{1}^{1}} P_{1}^{i} \longrightarrow \bigoplus_{i \in I_{0}-I_{0}^{1}} P_{0}^{i} \longrightarrow \frac{M}{M_{1}} \longrightarrow 0
$$

which is exact since the class of exact complexes is thick. Hence, we have a projective resolution of length $n$ for $M / M_{1}$. The rest of the proof follows by using an argument similar to the proof of Proposition 3.25.

Lemma 5.5. If $R$ is a Noetherian ring, then every chain complex in $\mathrm{dw} \widetilde{\mathcal{P}_{n}}$ has a dw $\widetilde{\mathcal{P}_{n}^{\aleph_{0}}}$-filtration.

Proof. Let

$$
X=\left(\cdots \longrightarrow X_{k+1} \stackrel{\partial_{k+1}}{\longrightarrow} X_{k} \stackrel{\partial_{k}}{\longrightarrow} X_{k-1} \longrightarrow \cdots\right)
$$


be a complex in $\mathrm{dw} \widetilde{\mathcal{P}_{n}}$. For each $k$, one has a projective resolution of $X_{k}$ of length $n$ :

$$
0 \longrightarrow \bigoplus_{i \in I_{n}(k)} P_{n}^{i}(k) \longrightarrow \cdots \longrightarrow \bigoplus_{i \in I_{0}(k)} P_{0}^{i}(k) \longrightarrow X_{k} \longrightarrow 0,
$$

where the modules appearing in the direct sums are countably generated and projective. It suffices to construct a nonzero subcomplex $X^{\prime} \subseteq X$ such that each $X_{k}^{\prime}$ has a projective resolution:

$$
0 \longrightarrow \bigoplus_{i \in I_{n}^{\prime}(k)} P_{n}^{i}(k) \longrightarrow \cdots \longrightarrow \bigoplus_{i \in I_{0}^{\prime}(k)} P_{0}^{i}(k) \longrightarrow X_{k}^{\prime} \longrightarrow 0
$$

where $I_{i}^{\prime}(k)$ is countable for every $0 \leq i \leq n$, and such that $X / X^{\prime} \in$ $\operatorname{dw} \widetilde{\mathcal{P}_{n}}$.

Fix $m \in \mathbb{Z}$. Let $S$ be a countably generated submodule of $X_{m}$. By Lemma 5.5, there exists a submodule

$$
\mathcal{P}_{n}^{\aleph_{0}} \ni X_{m}^{\prime} \subseteq X_{m}
$$

such that $S \subseteq X_{m}^{\prime}$. Note that $X_{m}^{\prime}$ is also countably generated. Then $\partial_{m}\left(X_{m}^{\prime}\right)$ is a countably generated submodule of $X_{m-1}$, and so there exists

$$
\mathcal{P}_{n}^{\aleph_{0}} \ni X_{m-1}^{\prime} \subseteq X_{m-1}
$$

such that $\partial_{m}\left(X_{m}^{\prime}\right) \subseteq X_{m-1}^{\prime}$. Repeat the same procedure infinitely many times in order to obtain a subcomplex

$$
X^{\prime}=\cdots \longrightarrow X_{k+1}^{\prime} \longrightarrow X_{k}^{\prime} \longrightarrow X_{k-1}^{\prime} \longrightarrow \cdots
$$

of $X$ such that $X_{k}^{\prime} \in \mathcal{P}_{n}^{\aleph_{0}}$ for every $k \in \mathbb{Z}$ (we are setting $X_{k}^{\prime}=0$ for every $k>m$ ). Hence, $X^{\prime} \in \mathrm{dw} \widetilde{\mathcal{P}_{n}^{\aleph_{0}}}$. Note from the proof of the previous lemma that the quotient $X / X^{\prime}$ is in $\mathrm{dw} \widetilde{\mathcal{P}_{n}}$. We have, for every $k \leq m$, the following projective resolutions of length $n$ for $X_{k} / X_{k}^{\prime}$ :

$$
0 \longrightarrow \bigoplus_{i \in I_{n}(k)-I_{n}^{\prime}(k)} P_{n}^{i}(k) \longrightarrow \cdots \longrightarrow \bigoplus_{i \in I_{0}(k)-I_{0}^{\prime}(k)} P_{0}^{i}(k) \longrightarrow \frac{X_{k}}{X_{k}^{\prime}} \longrightarrow 0
$$

The rest of the proof follows by transfinite induction.

It follows by Lemma 5.5 and Proposition 2.5 that the pair

$$
\left(\operatorname{dw} \widetilde{\mathcal{P}_{n}},\left(\operatorname{dw} \widetilde{\mathcal{P}_{n}}\right)^{\perp}\right)
$$


is complete and cogenerated by a set of representatives of the class $\mathrm{dw} \widetilde{\mathcal{P}_{n}^{\aleph_{0}}}$, provided $R$ is a Noetherian ring. Under this hypothesis, it is easy to show that the pair

$$
\left(\operatorname{ex} \widetilde{\mathcal{P}_{n}},\left(\operatorname{ex} \widetilde{\mathcal{P}_{n}}\right)^{\perp}\right)
$$

is also complete using Proposition 4.3, as long as we show that it is compatible with $\left(\mathrm{dw} \widetilde{\mathcal{P}_{n}},\left(\mathrm{dw} \widetilde{\mathcal{P}_{n}}\right)^{\perp}\right)$.

We know by [9, Theorem 3.12] that the cotorsion pairs

$$
(\operatorname{dg} \widetilde{\mathcal{A}}, \widetilde{\mathcal{B}}) \quad \text { and }(\widetilde{\mathcal{A}}, \operatorname{dg} \widetilde{\mathcal{B}})
$$

are compatible if the inducing pair $(\mathcal{A}, \mathcal{B})$ is hereditary. The author does not know if the same holds for the cotorsion pairs $\left(\mathrm{dw} \widetilde{\mathcal{A}},(\mathrm{dw} \widetilde{\mathcal{A}})^{\perp}\right)$ and $\left(\operatorname{ex} \widetilde{\mathcal{A}},(\operatorname{ex} \widetilde{\mathcal{A}})^{\perp}\right)$.

Consider the case $\mathcal{C}={ }_{R} \operatorname{Mod}$. Since

$$
\operatorname{dg} \widetilde{\mathcal{P}_{0}(\mathcal{C})} \subseteq \operatorname{dw} \widetilde{\mathcal{A}} \text { and } \quad \text { ex } \widetilde{\mathcal{A}} \subseteq \operatorname{dw} \widetilde{\mathcal{A}}
$$

we have

$$
(\mathrm{dw} \widetilde{\mathcal{A}})^{\perp} \subseteq\left(\operatorname{dg} \widetilde{\mathcal{P}_{0}(\mathcal{C})}\right)^{\perp}=\mathcal{E} \quad \text { and } \quad(\operatorname{dw} \widetilde{\mathcal{A}})^{\perp} \subseteq(\operatorname{ex} \widetilde{\mathcal{A}})^{\perp},
$$

and so

$$
(\mathrm{dw} \widetilde{\mathcal{A}})^{\perp} \subseteq(\operatorname{ex} \widetilde{\mathcal{A}})^{\perp} \cap \mathcal{E} .
$$

Similarly,

$$
{ }^{\perp}(\operatorname{dw} \widetilde{\mathcal{B}}) \subseteq{ }^{\perp}(\operatorname{ex} \widetilde{\mathcal{B}}) \cap \mathcal{E} .
$$

The next result provides a relationship between completeness of these degreewise cotorsion pairs and the remaining inclusions.

Proposition 5.6. Let $(\mathcal{A}, \mathcal{B})$ be a cotorsion pair in $\mathbf{C h}\left({ }_{R} \mathbf{M o d}\right)$.

(i) If the pair $\left(\mathrm{dw} \widetilde{\mathcal{A}},(\mathrm{dw} \widetilde{\mathcal{A}})^{\perp}\right)$ is complete, then

$$
(\operatorname{dw} \widetilde{\mathcal{A}})^{\perp}=(\operatorname{ex} \widetilde{\mathcal{A}})^{\perp} \cap \mathcal{E} .
$$

(ii) If the pair $\left({ }^{\perp}(\mathrm{dw} \widetilde{\mathcal{B}}), \mathrm{dw} \widetilde{\mathcal{B}}\right)$ is complete, then

$$
{ }^{\perp}(\operatorname{dw} \widetilde{\mathcal{B}})={ }^{\perp}(\operatorname{ex} \widetilde{\mathcal{B}}) \cap \mathcal{E} .
$$


Proof. We only prove the first statement. By the comments above, it suffices to show that

$$
(\operatorname{ex} \widetilde{\mathcal{A}})^{\perp} \cap \mathcal{E} \subseteq(\operatorname{dw} \widetilde{\mathcal{A}})^{\perp} .
$$

Let $Y \in(\operatorname{ex} \widetilde{\mathcal{A}})^{\perp} \cap \mathcal{E}$. Since $\left(\operatorname{dw} \widetilde{\mathcal{A}},(\operatorname{dw} \widetilde{\mathcal{A}})^{\perp}\right)$ is complete, there is a short exact sequence

$$
0 \longrightarrow Y \longrightarrow X \longrightarrow A \longrightarrow 0
$$

with $X \in(\operatorname{dw} \widetilde{\mathcal{A}})^{\perp}$ and $A \in \operatorname{dw} \widetilde{\mathcal{A}}$. Note that $A \in \operatorname{dw} \widetilde{\mathcal{A}} \cap \mathcal{E}$, since $X \in(\operatorname{dw} \widetilde{\mathcal{A}})^{\perp} \subseteq \mathcal{E}, Y \in \mathcal{E}$ and $\mathcal{E}$ is thick. It follows that $\operatorname{Ext}^{1}(A, Y)=0$ and that the previous sequence splits. We have that $Y$ is a direct summand of $X \in(\operatorname{dw} \widetilde{\mathcal{A}})^{\perp}$, and hence, $Y \in(\operatorname{dw} \widetilde{\mathcal{A}})^{\perp}$ since $(\operatorname{dw} \widetilde{\mathcal{A}})^{\perp}$ is closed under direct summands.

The previous result implies that the pairs

$$
\left(\operatorname{dw} \widetilde{\mathcal{P}_{n}},\left(\operatorname{dw} \widetilde{\mathcal{P}_{n}}\right)^{\perp}\right) \text { and }\left(\operatorname{ex} \widetilde{\mathcal{P}_{n}},\left(\operatorname{ex} \widetilde{\mathcal{P}_{n}}\right)^{\perp}\right)
$$

are compatible, and so $\left(\operatorname{ex} \widetilde{\mathcal{P}_{n}},\left(\operatorname{ex} \widetilde{\mathcal{P}_{n}}\right)^{\perp}\right)$ is complete, provided $R$ is a Noetherian ring. Using Hovey's correspondence, Theorem 5.1 follows. Note in its statement that we are not assuming $R$ to be Noetherian. In fact, it is possible to give a proof of this result that does not depend on this assumption. Such a proof consists of constructing, for every complex in ex $\widetilde{\mathcal{P}_{n}}$, a filtration by the class $\left(\right.$ ex $\left.\widetilde{\mathcal{P}_{n}}\right) \leq \kappa$, for a fixed infinite cardinal $\kappa>\operatorname{Card}(R)$.

Given a projective module $P$ written as a direct sum decomposition of countably generated projective modules $P=\bigoplus_{i \in I} P_{i}$, note that $P$ is $\kappa$-small if, and only if, Card $(I) \leq \kappa$.

\section{Definition 5.7.}

(i) Given $M \in \mathcal{P}_{n}$ with a projective resolution,

$$
(*)=\left(0 \longrightarrow \bigoplus_{i \in I_{n}} P_{n}^{i} \longrightarrow \cdots \longrightarrow \bigoplus_{i \in I_{1}} P_{1}^{i} \longrightarrow \bigoplus_{i \in I_{0}} P_{0}^{i} \longrightarrow M \longrightarrow 0\right)
$$

we shall say that a projective resolution

$$
(* *)=\left(0 \longrightarrow \bigoplus_{i \in I_{n}^{\prime}} P_{n}^{i} \longrightarrow \cdots \longrightarrow \bigoplus_{i \in I_{1}^{\prime}} P_{1}^{i} \longrightarrow \bigoplus_{i \in I_{0}^{\prime}} P_{0}^{i} \longrightarrow N \longrightarrow 0\right)
$$


is a nice subresolution of $(*)$ if $(* *)$ is a subcomplex of $(*)$ such that $I_{k}^{\prime} \subseteq I_{k}$ for every $0 \leq k \leq n$.

Let $\kappa$ be an infinite cardinal satisfying $\kappa>\operatorname{Card}(R)$.

(ii) We shall denote by $\mathcal{P}_{n}(\kappa)$ the class of $n$-projective modules $M$ with a projective resolution $(*)$ such that Card $\left(I_{k}\right) \leq \kappa$ for every $0 \leq k \leq n$. Note that $\mathcal{P}_{n}(\kappa) \subseteq\left(\mathcal{P}_{n}\right) \leq \kappa$.

(iii) If we consider the resolutions $(*)$ and $(* *)$ above, then we say that $(* *)$ is a nice $\kappa$-small subresolution of $(*)$ if each $I_{k}^{\prime}$ is a $\kappa$-small subset of $I_{k}$.

Lemma 5.8. Let $\kappa$ be an infinite regular cardinal satisfying $\kappa>$ Card $(R)$. Let $M \in \mathcal{P}_{n}$ with a projective resolution be given by $(*)$. For every $\kappa$-small submodule $N \subseteq M$, there exists a nice $\kappa$-small subresolution

$(* * *)=\left(0 \longrightarrow \bigoplus_{i \in I_{n}^{\prime \prime}} P_{n}^{i} \longrightarrow \cdots \longrightarrow \bigoplus_{i \in I_{1}^{\prime \prime}} P_{1}^{i} \longrightarrow \bigoplus_{i \in I_{0}^{\prime \prime}} P_{0}^{i} \longrightarrow N^{\prime} \longrightarrow 0\right)$

of $(*)$ such that $N \subseteq N^{\prime}$. Moreover, if $N$ has a nice $\kappa$-small subresolution $(* *)$ of $M$, then $(* * *)$ can be constructed in such a way that (**) is a subresolution of $(* * *)$.

Proof. Using the zigzag procedure in a similar way as in Proposition 5.4, it is possible to construct $N^{\prime}$ as described in Lemma 5.8. Now suppose that $N$ has a nice $\kappa$-small subresolution

$$
(* *)=\left(0 \longrightarrow \bigoplus_{i \in I_{n}^{\prime}} P_{n}^{i} \longrightarrow \cdots \longrightarrow \bigoplus_{i \in I_{1}^{\prime}} P_{1}^{i} \longrightarrow \bigoplus_{i \in I_{0}^{\prime}} P_{0}^{i} \longrightarrow N \longrightarrow 0\right)
$$

of $(*)$. Take the quotient of $(*)$ by this resolution and get

$$
0 \longrightarrow \bigoplus_{i \in I_{n}-I_{n}^{\prime}} P_{n}^{i} \longrightarrow \cdots \longrightarrow \bigoplus_{i \in I_{1}-I_{1}^{\prime}} P_{1}^{i} \longrightarrow \bigoplus_{i \in I_{0}-I_{0}^{\prime}} P_{0}^{i} \longrightarrow \frac{M}{N} \longrightarrow 0 .
$$

Consider the $\kappa$-small submodule $\langle z+N\rangle$, with $z \notin N$, and apply the zigzag procedure to get a projective subresolution of the previous one,

$$
0 \longrightarrow \bigoplus_{i \in I_{n}^{\prime \prime}-I_{n}^{\prime}} P_{n}^{i} \longrightarrow \cdots \longrightarrow \bigoplus_{i \in I_{1}^{\prime \prime}-I_{1}^{\prime}} P_{1}^{i} \longrightarrow \bigoplus_{i \in I_{0}^{\prime \prime}-I_{0}^{\prime}} P_{0}^{i} \longrightarrow \frac{N^{\prime}}{N} \longrightarrow 0
$$


where each set $I_{k}^{\prime \prime}-I_{k}^{\prime}$ is a $\kappa$-small set. Finally, note that

$$
(* * *)=\left(0 \longrightarrow \bigoplus_{i \in I_{n}^{\prime \prime}} P_{n}^{i} \longrightarrow \cdots \longrightarrow \bigoplus_{i \in I_{1}^{\prime \prime}} P_{1}^{i} \longrightarrow \bigoplus_{i \in I_{0}^{\prime \prime}} P_{0}^{i} \longrightarrow N^{\prime} \longrightarrow 0\right)
$$

is a nice $\kappa$-small subresolution of $(*)$ containing $(* *)$.

Lemma 5.9. Let $\kappa$ be an infinite regular cardinal satisfying $\kappa>$ Card $(R)$. Let $X \in \mathrm{dw} \widetilde{\mathcal{P}_{n}}$ and $Y$ a $\kappa$-small and bounded above subcomplex of $X$. Then there exists a (bounded above) subcomplex $Y^{\prime}$ of $X$ such that $Y \subseteq Y^{\prime}$ and $Y^{\prime} \in \operatorname{dw} \widehat{\mathcal{P}_{n}(\kappa)}$.

Proof. Since $Y$ is a complex bounded from above, there exists $m \in \mathbb{Z}$ such that $Y_{k}=0$ for every $k>m$. We obtain the following commutative diagram where the vertical arrows are monomorphisms:

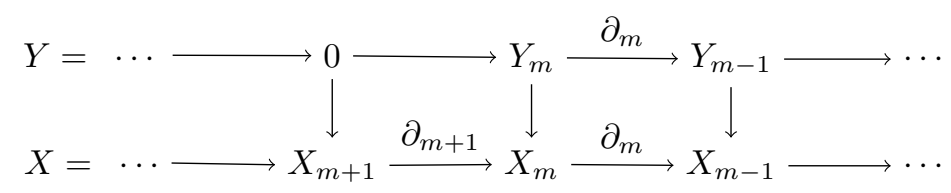

Since $X_{m}$ is an $n$-projective module, we have a projective resolution

$$
0 \longrightarrow \bigoplus_{i \in I_{n}(m)} P_{n}^{i}(m) \longrightarrow \cdots \longrightarrow \bigoplus_{i \in I_{0}(m)} P_{0}^{i}(m) \longrightarrow X_{m} \longrightarrow 0
$$

By Lemma 5.9, there exists a submodule $Y_{m}^{\prime}$ of $X_{m}$ containing $Y_{m}$, along with a nice $\kappa$-small subresolution

$$
0 \longrightarrow \bigoplus_{i \in I_{n}^{\prime}(m)} P_{n}^{i}(m) \longrightarrow \cdots \longrightarrow \bigoplus_{i \in I_{0}^{\prime}(m)} P_{0}^{i}(m) \longrightarrow Y_{m}^{\prime} \longrightarrow 0
$$

Note that

$$
\operatorname{Card}\left(\partial_{m}\left(Y_{m}^{\prime}\right)+Y_{m-1}\right) \leq \kappa
$$

and

$$
Y_{m-1} \subseteq \partial_{m}\left(Y_{m}^{\prime}\right)+Y_{m-1} \subseteq X_{m-1} .
$$

Now choose a submodule $Y_{m-1}^{\prime} \subseteq X_{m-1}$ with

$$
\partial_{m}\left(Y_{m}^{\prime}\right)+Y_{m-1} \subseteq Y_{m-1}^{\prime}
$$


and $Y_{m-1}^{\prime}$ has a nice $\kappa$-small subresolution of a fixed resolution of $X_{m-1}$. Repeat this process infinitely many times in order to obtain a chain complex,

$$
Y^{\prime}=\cdots \longrightarrow 0 \longrightarrow Y_{m}^{\prime} \longrightarrow Y_{m-1}^{\prime} \longrightarrow \cdots \quad \text { in } \mathrm{dw} \widetilde{\mathcal{P}_{n}(\kappa)},
$$

such that $Y \subseteq Y^{\prime} \subseteq X$.

Theorem 5.10. Every complex $X \in \mathrm{ex} \widetilde{\mathcal{P}_{n}}$ has an ex $\widetilde{\mathcal{P}_{n}(\kappa)}$-filtration.

Proof. Let $m \in \mathbb{Z}$ be arbitrary and $T_{1} \subseteq X_{m}$ a $\kappa$-small submodule of $X_{m}$. Fix, for $X_{m}$, a projective resolution of length $n$. By Lemma 5.8, there exists a $\kappa$-small submodule $Y_{m}^{1}$ of $X_{m}$ such that $T_{1} \subseteq Y_{m}^{1}$ and $Y_{m}^{1}$ has a nice $\kappa$-small projective subresolution of the given resolution of $X_{m}$. Note that $\partial_{m}\left(Y_{m}^{1}\right)$ is a $\kappa$-small submodule of $X_{m-1}$, so there exists a submodule $Y_{m-1}^{1}$ of $X_{m-1}$ such that $\partial_{m}\left(Y_{m}^{1}\right) \subseteq Y_{m-1}^{1}$ and $Y_{m-1}^{1}$ has a nice $\kappa$-small projective subresolution of the given resolution of $X_{m-1}$. Keep repeating this argument infinitely many times. We obtain a subcomplex,

$$
Y^{1}=\left(\cdots \longrightarrow 0 \longrightarrow Y_{m}^{1} \longrightarrow Y_{m-1}^{1} \longrightarrow \cdots\right) \subseteq X,
$$

in $\mathrm{dw} \widetilde{\mathcal{P}_{n}(\kappa)}$. Note that $Y^{1}$ is not necessarily exact.

We shall construct a complex $X^{1}$ from $Y^{1}$ such that

$$
X^{1} \subseteq X \quad \text { and } \quad X^{1} \in \operatorname{ex} \widetilde{\mathcal{P}_{n}(\kappa)} .
$$

The rest of this proof uses an argument similar to the one in $[4$, Theorem 4.6]. Fix any $p \in \mathbb{Z}$. Then $\operatorname{Card}\left(Y_{p}^{1}\right) \leq \kappa$ and so $\operatorname{Card}\left(Z_{p}\left(Y^{1}\right)\right) \leq$ $\kappa$. Since $X$ is exact and $\operatorname{Card}\left(Z_{p}\left(Y^{1}\right)\right) \leq \kappa$, there exists a submodule $U \subseteq X_{p+1}$ with $\operatorname{Card}(U) \leq \kappa$ such that

$$
Z_{p}\left(Y^{1}\right) \subseteq \partial_{p+1}(U)
$$

Let $C^{1}$ be a $\kappa$-small subcomplex of $X$ such that

$$
U \subseteq C_{p+1}, \quad C_{j}=0 \text { for every } j>p+1,
$$

and such that each $C_{j}$ with $j \leq p$ has a nice $\kappa$-small projective subresolution of a given resolution of $X_{j}$. Since $Y^{1}+C$ is a subcomplex bounded above by $X$, by Lemma 5.2 there exists a $\kappa$-small subcomplex $Y^{2}$ of $X$ such that $Y^{1}+C \subseteq Y^{2}$ and such that each $Y_{j}^{2}$ has a nice 
$\kappa$-small projective subresolution of a given resolution of $X_{j}$. Note that $Z_{p}\left(Y^{1}\right) \subseteq \partial_{p+1}\left(Y_{p+1}^{2}\right)$.

Construct $Y^{3}$ from $Y^{2}$ as $Y^{2}$ was constructed from $Y^{1}$, and so on, making sure to use the same $p \in \mathbb{Z}$ at each step. Set

$$
X^{1}=\bigcup_{j=1}^{\infty} Y^{j} \subseteq X
$$

Note that $X^{1}$ is exact at $p$. Repeat this argument to get exactness at any level. So we may assume that $X^{1}$ is an exact complex. Every $X_{k}^{1}$ has a nice $\kappa$-small projective subresolution of the given resolution of $X_{k}$ :

$$
0 \longrightarrow \bigoplus_{i \in I_{n}(k)} P_{n}^{i}(k) \longrightarrow \cdots \longrightarrow \bigoplus_{i \in I_{0}(k)} P_{0}^{i}(k) \longrightarrow X_{k} \longrightarrow 0 .
$$

Indeed, for every $j$, one has a projective subresolution of the form

$$
0 \longrightarrow \bigoplus_{i \in I_{n}^{j}(k)} P_{n}^{i}(k) \longrightarrow \cdots \longrightarrow \bigoplus_{i \in I_{0}^{j}(k)} P_{0}^{i}(k) \longrightarrow Y_{k}^{j} \longrightarrow 0,
$$

where

$$
I_{l}^{1}(k) \subseteq I_{l}^{2}(k) \subseteq \cdots \quad \text { for every } 0 \leq l \leq n,
$$

by Lemma 5.8. If we take the union of all of the previous sequences, we obtain the following exact sequence:

$$
0 \longrightarrow \bigoplus_{i \in \cup_{j \geq i} I_{n}^{j}(k)} P_{n}^{i}(k) \longrightarrow \cdots \longrightarrow \bigoplus_{i \in \cup_{j \geq 1} I_{0}^{j}(k)} P_{0}^{i}(k) \longrightarrow \bigcup_{j \geq 1} Y_{k}^{j} \longrightarrow 0,
$$

where

$$
\bigcup_{j \geq 1} I_{l}^{j}(k) \subseteq I_{l}(k) \quad \text { for every } 0 \leq l \leq n,
$$

and

$$
\bigcup_{j \geq 1} Y_{k}^{j}=X_{j}^{1} .
$$

Therefore, $X^{1} \in$ ex $\widetilde{\mathcal{P}_{n}(\kappa)}$. Moreover, note that $X / X^{1} \in \operatorname{ex} \widetilde{\mathcal{P}_{n}}$. The rest of the proof follows by using transfinite induction. 
It follows by Proposition 2.5 that $\left(\mathrm{ex} \widetilde{\mathcal{P}_{n}},\left(\mathrm{ex} \widetilde{\mathcal{P}_{n}}\right)^{\perp}\right)$ is a cotorsion pair cogenerated by a set of representatives of the class ex $\widetilde{\mathcal{P}_{n}(\kappa)}$, and so it is complete by Eklof and Trlifaj's theorem.

Degreewise $n$-projective model structures are not monoidal in general. However, we shall see at the end of the next section that the degreewise projective model structure is monoidal if $R$ is a commutative ring with weak dimension $\leq 1$.

We conclude this section by presenting the dual of the degreewise $n$-projective model structure. Consider the class $\mathcal{I}_{n}$ of $n$-injective modules.

Theorem 5.11. The two compatible and complete cotorsion pairs,

$$
(\mathcal{A}, \mathcal{B} \cap \mathcal{W})=\left({ }^{\perp}\left(\operatorname{ex} \widetilde{\mathcal{I}_{n}}\right), \operatorname{ex} \widetilde{\mathcal{I}_{n}}\right)
$$

and

$$
(\mathcal{A} \cap \mathcal{W}, \mathcal{B})=\left({ }^{\perp}\left(\mathrm{dw} \widetilde{\mathcal{I}_{n}}\right), \mathrm{dw} \widetilde{\mathcal{I}_{n}}\right),
$$

give rise to a unique Abelian model category structure on $\mathbf{C h}\left({ }_{R} \mathbf{M o d}\right)$, called the degreewise $n$-injective model structure, such that $\mathcal{A}=\perp$ $\left(\operatorname{ex} \widetilde{\mathcal{I}_{n}}\right), \mathcal{B}=\operatorname{dw} \widetilde{\mathcal{I}_{n}}$ and $\mathcal{W}=\mathcal{E}$ are the classes of cofibrant, fibrant and trivial objects, respectively.

Given a Grothendieck category $\mathcal{C}$ with a generator $G$, consider the class $\mathcal{S}$ of all disk complexes $D^{m}(C)$ with $C \in \Omega^{n}(G / J)$ and $J$ running over the set of subobjects of $G$. If $D^{m}(C) \in \mathcal{S}$ and $Y \in \mathrm{dw} \widetilde{\mathcal{I}_{n}(\mathcal{C})}$, using $[\mathbf{9}$, Lemma $3.1(5)]$ we have

$$
\operatorname{Ext}_{\mathbf{C h}(\mathcal{C})}^{1}\left(D^{m}(C), Y\right) \cong \operatorname{Ext}_{\mathcal{C}}^{1}\left(C, Y_{m}\right) .
$$

On the other hand,

$$
\operatorname{Ext}_{\mathcal{C}}^{1}\left(C, Y_{m}\right) \cong \operatorname{Ext}_{\mathcal{C}}^{n+1}\left(G / J, Y_{m}\right)=0,
$$

since $Y_{m}$ is $n$-injective. Then, $D^{m}(C) \in{ }^{\perp}\left(\operatorname{dw} \widetilde{\mathcal{I}_{n}(\mathcal{C})}\right)$. This implies $\mathrm{dw} \widetilde{\mathcal{I}_{n}(\mathcal{C})} \subseteq \mathcal{S}^{\perp}$. 
The other inclusion follows in the same way, and so dw $\widetilde{\mathcal{I}_{n}(\mathcal{C})}=\mathcal{S}^{\perp}$. It follows that $\left({ }^{\perp}\left(\mathrm{dw} \widetilde{\mathcal{I}_{n}}\right), \mathrm{dw} \widetilde{\mathcal{I}_{n}}\right)$ is complete. By Propositions $4.3,5.6$ and Hovey's correspondence, Theorem 5.11 follows.

6. $n$-flat and degreewise $n$-flat model structures. For $n>0$, let $\mathcal{F}_{n}$ denote the class of left $n$ - $\mathcal{F}_{0}$-modules of ${ }_{R} \mathbf{M o d}$, where $\mathcal{F}_{0}$ denotes the class of flat modules. We shall say that $M \in \mathrm{Ob}\left({ }_{R} \mathbf{M o d}\right)$ is $n$-flat if $M \in \mathcal{F}_{n}$. It is known that $\left(\mathcal{F}_{n},\left(\mathcal{F}_{n}\right)^{\perp}\right)$ is a hereditary and complete cotorsion pair (see [11, Theorem 4.1.3]). It follows by [9, Corollary 3.8, Theorem 3.12] that we have two compatible cotorsion pairs

$$
\left(\widetilde{\mathcal{F}_{n}},\left(\widetilde{\mathcal{F}_{n}}\right)^{\perp}\right) \text { and }\left(\operatorname{dg} \widetilde{\mathcal{F}_{n}},\left(\operatorname{dg} \widetilde{\mathcal{F}_{n}}\right)^{\perp}\right) .
$$

On the other hand, by [10, Propositions 3.2, 3.3], we have two cotorsion pairs

$$
\left(\operatorname{dw} \widetilde{\mathcal{F}_{n}},\left(\operatorname{dw} \widetilde{\mathcal{F}_{n}}\right)^{\perp}\right) \text { and }\left(\operatorname{ex} \widetilde{\mathcal{F}_{n}},\left(\operatorname{ex} \widetilde{\mathcal{F}_{n}}\right)^{\perp}\right) .
$$

The goal of this section is to construct filtrations for the classes $\widetilde{\mathcal{F}_{n}}$ and ex $\widetilde{\mathcal{F}_{n}}$ by the sets $\left(\widetilde{\mathcal{F}_{n}}\right) \leq \kappa$ and $\left(\right.$ ex $\left.\widetilde{\mathcal{F}_{n}}\right) \leq \kappa$, respectively, based on early investigations developed by Aldrich, et al., [1] for the case $n=0$. After obtaining those results, we deduce the completeness of the previous pairs and then the existence of the following two model structures:

Theorem 6.1. The two compatible and complete cotorsion pairs,

$$
(\mathcal{A}, \mathcal{B} \cap \mathcal{W})=\left(\operatorname{dg} \widetilde{\mathcal{F}_{n}},\left(\operatorname{dg} \widetilde{\mathcal{F}_{n}}\right)^{\perp}\right)
$$

and

$$
(\mathcal{A} \cap \mathcal{W}, \mathcal{B})=\left(\widetilde{\mathcal{F}_{n}},\left(\widetilde{\mathcal{F}_{n}}\right)^{\perp}\right),
$$

give rise to a unique Abelian model category structure on $\mathbf{C h}\left({ }_{R} \mathbf{M o d}\right)$, called the $n$-flat model structure, such that $\mathcal{A}=\operatorname{dg} \widetilde{\mathcal{F}_{n}}, \mathcal{B}=\left(\widetilde{\mathcal{F}_{n}}\right)^{\perp}$ and $\mathcal{W}=\mathcal{E}$ are the classes of cofibrant, fibrant and trivial objects, respectively.

Theorem 6.2. The two compatible and complete cotorsion pairs,

$$
(\mathcal{A}, \mathcal{B} \cap \mathcal{W})=\left(\operatorname{dw} \widetilde{\mathcal{F}_{n}},\left(\operatorname{dw} \widetilde{\mathcal{F}_{n}}\right)^{\perp}\right)
$$


and

$$
(\mathcal{A} \cap \mathcal{W}, \mathcal{B})=\left(\operatorname{ex} \widetilde{\mathcal{F}_{n}},\left(\operatorname{ex} \widetilde{\mathcal{F}_{n}}\right)^{\perp}\right)
$$

give rise to a unique Abelian model category structure on $\mathbf{C h}\left({ }_{R} \mathbf{M o d}\right)$, called the degreewise $n$-flat model structure, such that $\mathcal{A}=\operatorname{dw} \widetilde{\mathcal{F}_{n}}$, $\mathcal{B}=\left(\operatorname{ex} \widetilde{\mathcal{F}_{n}}\right)^{\perp}$ and $\mathcal{W}=\mathcal{E}$ are the classes of cofibrant, fibrant and trivial objects, respectively.

For the case $n=0$, the completeness of the pairs

$$
\left(\widetilde{\mathcal{F}_{0}},\left(\widetilde{\mathcal{F}_{0}}\right)^{\perp}\right) \quad \text { and } \quad\left(\operatorname{dg} \widetilde{\mathcal{F}_{0}},\left(\operatorname{dg} \widetilde{\mathcal{F}_{0}}\right)^{\perp}\right)
$$

was proven by Gillespie in [9], using the notions of pure and dgpure subcomplexes. The compatibility of these two pairs and Hovey's correspondence allowed Gillespie to find a unique monoidal Abelian model structure on $\mathbf{C h}\left({ }_{R}\right.$ Mod), called the flat model structure, where the weak equivalences are quasi-isomorphisms, the (trivial) cofibrations are injections with (exact) dg-flat cokerels and the (trivial) fibrations are surjections with (exact) dg-cotorsion kernels (see [9, Corollary 5.1]).

The cotorsion pair $\left(\mathcal{F}_{0},\left(\mathcal{F}_{0}\right)^{\perp}\right)$ was proven to be complete by Enochs. His argument consists of showing that every flat module is filtered by the class of $\kappa$-small flat modules, with $\kappa$ an infinite regular cardinal satisfying $\kappa>$ Card $(R)$. Enoch's arguments allow us to construct filtrations for the class $\mathcal{F}_{n}$ by the class $\left(\mathcal{F}_{n}\right) \leq \kappa$. Regarding this matter, Lemma 6.4 shall help us to construct filtrations for $\widetilde{\mathcal{F}_{n}}$ and ex $\widetilde{\mathcal{F}_{n}}$, mentioned above.

Definition 6.3. Recall that a submodule $S$ of a left $R$-module $M$ is pure if the sequence $0 \rightarrow N \otimes_{R} S \rightarrow N \otimes_{R} M$ is exact, for every right $R$-module $N$. If $M$ is an $n$-flat module and $N \subseteq M$ is a submodule, we say that $N$ is an $n$-pure submodule of $M$ if there exist an exact sequence,

$$
0 \longrightarrow F_{n} \longrightarrow \cdots \longrightarrow F_{1} \longrightarrow F_{0} \longrightarrow M \longrightarrow 0,
$$

with each $F_{k}$ flat and a commutative diagram with exact rows 


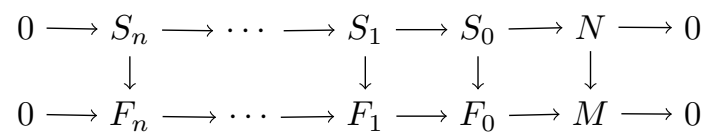

such that each $S_{k} \rightarrow F_{k}$ is an inclusion with $S_{k}$ a pure submodule.

Lemma 6.4. Let $\kappa$ be an infinite regular cardinal satisfying $\kappa>$ Card $(R), M \in \mathcal{F}_{n}$, with a flat resolution

$$
(1)=\left(0 \longrightarrow F_{n} \stackrel{f_{n}}{\longrightarrow} F_{n-1} \longrightarrow \cdots \longrightarrow F_{1} \stackrel{f_{1}}{\longrightarrow} F_{0} \stackrel{f_{0}}{\longrightarrow} M \longrightarrow 0\right),
$$

and let $N$ be a $\kappa$-small submodule of $M$. Then there exists an n-pure submodule $N^{\prime}$ of $M$ containing $N$ with a resolution,

$$
0 \longrightarrow S_{n}^{\prime} \longrightarrow \cdots \longrightarrow S_{1}^{\prime} \longrightarrow S_{0}^{\prime} \longrightarrow N^{\prime} \longrightarrow 0,
$$

such that $S_{k}^{\prime}$ is a $\kappa$-small and pure submodule of $F_{k}$, for every $0 \leq k \leq$ $n$. Moreover, if $N$ is an n-pure submodule of $M$ with a resolution,

$$
0 \longrightarrow S_{n} \longrightarrow \cdots \longrightarrow S_{1} \longrightarrow S_{0} \longrightarrow N \longrightarrow 0,
$$

as in Definition 6.3, then the above resolution of $N^{\prime}$ can be constructed in such a way that it contains the given resolution of $N$.

Proof. For every $x \in N$, there exists $y_{x} \in F_{0}$ such that $x=f_{0}\left(y_{x}\right)$. Consider the set

$$
Y:=\left\{y_{x}: x \in N \text { and } f_{0}\left(y_{x}\right)=x\right\}
$$

and the submodule $\langle Y\rangle \subseteq F_{0}$. Since $\langle Y\rangle$ is $\kappa$-small, there exists a $\kappa$-small pure submodule $S_{0}(1) \subseteq F_{0}$ such that $\langle Y\rangle \subseteq S_{0}(1)$, by [7, Lemma 5.3.12]. Note that $f_{0}\left(S_{0}(1)\right) \supseteq N$. The rest of the proof follows by applying the zigzag procedure as in the proof of Lemma 3.24. Next, we have for each $0 \leq k \leq n$ a collection of modules $\left(S_{k}(i): i \geq 0\right)$ such that each $S_{k}(i)$ is a pure submodule of $F_{k}$ and such that

$$
(2)=\left(0 \longrightarrow S_{n}^{\prime} \longrightarrow S_{n-1}^{\prime} \longrightarrow \cdots \longrightarrow S_{1}^{\prime} \longrightarrow S_{0}^{\prime} \longrightarrow N^{\prime} \longrightarrow 0\right),
$$

is exact, where

$$
S_{k}^{\prime}=\bigcup_{i \geq 0} S_{k}(i)
$$


is a $\kappa$-small and pure submodule of $F_{k}$ (and so it is flat) and $Q=$ $\operatorname{CoKer}\left(\left.f_{1}\right|_{S_{1}^{\prime}}\right) \subseteq M$. If we take the quotient of (1) by (2), we get a flat resolution of $M / N^{\prime}$ of length $n$ (note that the quotient of a flat module by a pure submodule is also flat). The rest of the statement follows as in Lemma 5.8.

Regarding the class $\mathcal{F}_{n}$, the pair $\left(\mathcal{F}_{n},\left(\mathcal{F}_{n}\right)^{\perp}\right)$ is perfect. A cotorsion pair $(\mathcal{A}, \mathcal{B})$ in an Abelian category $\mathcal{C}$ is said to be perfect if $\mathcal{A}$ is a covering class and $\mathcal{B}$ is an enveloping class, i.e., every module has an $\mathcal{A}$-cover and a $\mathcal{B}$-envelope. A map $f: A \rightarrow X$ is said to be an $\mathcal{A}$-cover of $X$ if $A \in \mathcal{A}$ and if the following conditions are satisfied:

(1) If $f^{\prime}: A^{\prime} \rightarrow X$ is another map with $A^{\prime} \in \mathcal{A}$, then there exists a map $h: A^{\prime} \rightarrow A$ (not necessarily unique) such that $f^{\prime}=f \circ h$.

(2) If $A^{\prime}=A$ and $f^{\prime}=f$ in (1), then every map $h: A \rightarrow A$ satisfying the equality $f^{\prime}=f \circ h$ is an automorphism of $A$.

The notion of $\mathcal{B}$-envelope is dual. In [11, Corollary 5.32], the authors prove that if $(\mathcal{A}, \mathcal{B})$ is a complete cotorsion pair such that $\mathcal{A}$ is closed under direct limits, then $(\mathcal{A}, \mathcal{B})$ is perfect. Since the bi-functors $\operatorname{Tor}_{i}^{R}(-,-)$ preserve filtered colimits, it follows that the class $\mathcal{F}_{n}$ is closed under direct limits, and hence, we can conclude that every left $R$-module has an $n$-flat cover (the case $n=0$ was known as the flat cover conjecture until 2000 when it was proven to be true by Bican, El Bashir and Enochs in [3]). We shall see that this result is also valid in the category $\mathbf{C h}\left({ }_{R} \mathbf{M o d}\right)$ of chain complexes of modules.

First, we need to show that $\left(\widetilde{\mathcal{F}_{n}},\left(\widetilde{\mathcal{F}}_{n}\right)^{\perp}\right)$ is complete. In $[\mathbf{8}$, Definition 4.1.2], flat chain complexes are defined as those complexes $F \in \mathrm{Ob}\left(\mathbf{C h}\left({ }_{R} \mathbf{M o d}\right)\right)$ such that $F$ is exact and $Z_{m}(F)$ is a flat module for every $m \in \mathbb{Z}$, or equivalently, those $F$ such that the functor $-\bar{\otimes} F: \mathbf{C h}\left(\mathbf{M o d}_{R}\right) \rightarrow \mathbf{A b}$ is left exact (see [8, Proposition 5.1.2]). As shown in Proposition 3.6, a chain complex $X$ is $n$-flat if, and only if, it is exact and $Z_{m}(X) \in \mathcal{F}_{n}$, for every $m \in \mathbb{Z}$.

In [1, Proposition 3.1], the authors prove that each element of a flat chain complex $F$ is contained in a $\kappa$-small flat subcomplex $L \subseteq F$ such that the quotient $F / L$ is also flat. Using the procedure in [1], along with Lemma 6.4, we obtain Theorem 6.5. 
Theorem 6.5. Let $\kappa$ be an infinite regular cardinal satisfying $\kappa>$ Card $(R)$. For any $n$-flat complex $X \in \widetilde{\mathcal{F}}_{n}$ and any element $x \in X$, i.e., $x \in X_{k}$ for some $k \in \mathbb{Z}$, there exists an exact subcomplex $L \subseteq X$ with $x \in L$ such that $Z_{m}(L)$ is a $k$-small and $n$-pure submodule of $Z_{m}(X)$, for every $m \in \mathbb{Z}$.

Note that if $L$ is the complex provided by the previous result, we have a short exact sequence $0 \rightarrow L \rightarrow X \rightarrow X / L \rightarrow 0$ in $\mathbf{C h}\left({ }_{R} \mathbf{M o d}\right)$. Note that $X / L$ is exact, since $L$ and $X$ are exact and the class of exact complexes is thick. By Lemma 3.5, we have the short exact sequence

$$
0 \longrightarrow Z_{n}(L) \longrightarrow Z_{n}(X) \longrightarrow Z_{n}(X / L) \longrightarrow 0 .
$$

It follows that

$$
Z_{n}(X / L) \cong Z_{n}(X) / Z_{n}(L),
$$

which is $n$-flat since $Z_{n}(X)$ is $n$-flat and $Z_{n}(L)$ is an $n$-pure submodule of $Z_{n}(X)$. Therefore, $X / L \in \widetilde{\mathcal{F}_{n}}$. By Proposition 3.25, $\widetilde{\mathcal{F}_{n}}$ is filtered by the class $\left(\widetilde{\mathcal{F}_{n}}\right) \leq \kappa$. So Propositions 2.5, 3.25 and Eklof and Trlijaf's theorem imply that the pair $\left(\widetilde{\mathcal{F}_{n}},\left(\widetilde{\mathcal{F}_{n}}\right)^{\perp}\right)$ is complete.

We have that

$$
\left(\operatorname{dg} \widetilde{\mathcal{F}_{n}} \cap \mathcal{E},\left(\widetilde{\mathcal{F}_{n}}\right)^{\perp}\right) \quad \text { and } \quad\left(\operatorname{dg} \widetilde{\mathcal{F}_{n}},\left(\widetilde{\mathcal{F}_{n}}\right)^{\perp} \cap \mathcal{E}\right)
$$

are compatible cotorsion pairs where the former is complete: by Proposition 4.3, the latter is also complete. Therefore, Theorem 6.1 follows. As in the $n$-projective model structure, note that we can use [9, Corollary 3.8] to describe the fibrant objects as those chain complexes $Y$ such that each $Y_{m}$ is right orthogonal to the class $\mathcal{F}_{n}$ and such that every chain map $X \rightarrow Y$ is homotopic to 0 whenever $X$ is an $n$-flat complex.

For $n=0$, the flat model structure is monoidal, as proved by Gillespie in [9, Corollary 5.1]. For $n>0$, the $n$-flat model structure is not monoidal in general. The same counterexample used for the projective case works.

Let $\overline{\operatorname{Tor}}_{i}(-,-)$ be the left derived functors of $-\bar{\otimes}-$. For every fixed chain complex $X \in \mathrm{Ob}\left(\mathbf{C h}\left(\boldsymbol{M o d}{ }_{R}\right)\right)$, the functor $\overline{\operatorname{Tor}}_{1}(X,-)$ preserves direct limits. This is a consequence of [8, Proposition 4.2.1 5]. It follows that the class $\widetilde{\mathcal{F}_{n}}$ is closed under direct limits, and hence the 
pair $\left(\widetilde{\mathcal{F}_{n}},\left(\widetilde{\mathcal{F}_{n}}\right)^{\perp}\right)$ is perfect by $[\mathbf{1 1}$, Corollary 5.32]. Hence, we obtain the following result.

Corollary 6.6. Every chain complex in $\mathbf{C h}\left({ }_{R} \mathbf{M o d}\right)$ has an $n$-flat cover.

The first approaches for complexes over a commutative Noetherian ring with finite Krull dimension are given in [8, subsections 4.3, 4.4] in the case $n=0$.

We finish this section by giving a characterization of pure subcomplexes of flat complexes. We recall from [9, Definition 4.3] that a complex $S$ in $\mathbf{C h}\left({ }_{R} \mathbf{M o d}\right)$ is a pure subcomplex of a complex $X$ if the sequence

$$
0 \longrightarrow Y \bar{\otimes} S \longrightarrow Y \bar{\otimes} X
$$

is exact, for every $Y \in \mathrm{Ob}\left(\mathbf{C h}\left(\mathbf{M o d}_{R}\right)\right)$.

Proposition 6.7. $S$ is a pure subcomplex of a flat complex $F$ if, and only if, $S$ is exact and $Z_{m}(S)$ is a pure submodule of $Z_{m}(F)$, for every $m \in \mathbb{Z}$

Proof. Suppose $S$ is a pure subcomplex of $\mathscr{F}$. Then $S$ is flat by [9, Lemma 4.7], and so it is exact. It suffices to show $Z_{m}(S)$ is a pure submodule of $Z_{m}(\mathscr{F})$. Let $M$ be a right $R$-module. Consider the sphere complex $S^{0}(M)$. Since $S$ is a pure subcomplex of $F$, the sequence

$$
0 \longrightarrow S^{0}(M) \bar{\otimes} S \longrightarrow S^{0}(M) \bar{\otimes} F
$$

is exact. At each $m \in \mathbb{Z}$, we have that $\left(S^{0}(M) \otimes X\right)_{m}=M \otimes_{R} X_{m}$, for every complex $X \in \mathrm{Ob}\left(\mathbf{C h}\left({ }_{R} \mathbf{M o d}\right)\right)$.

Recall the boundary map $\partial_{m+1}^{S^{0}(M) \otimes X}$ is given by

$$
y \otimes x \longmapsto y \otimes \partial_{m+1}^{X}(x)
$$

on generators. It is easy to see that

$$
M \otimes_{R} B_{m}(X)=B_{m}\left(S^{0}(M) \otimes X\right) .
$$

Then it follows that the maps

$$
M \otimes_{R} B_{m}(X) \longrightarrow M \otimes_{R} X_{m}
$$


are injective, so

$$
\left(S^{0}(M) \bar{\otimes} X\right)_{m}=\frac{\left(S^{0}(M) \otimes X\right)_{m}}{B_{m}\left(S^{0}(M) \otimes X\right)}=\frac{M \otimes_{R} X_{m}}{M \otimes_{R} B_{m}(X)} \cong M \otimes_{R} \frac{X_{m}}{B_{m}(X)} .
$$

Since $S$ and $F$ are exact, we get

$$
\left(S^{0}(M) \bar{\otimes} S\right)_{m} \cong M \otimes_{R} Z_{m-1}(S)
$$

and

$$
\left(S^{0}(M) \bar{\otimes} F\right)_{m} \cong M \otimes_{R} Z_{m-1}(F) .
$$

For every $m \in \mathbb{Z}$,

$$
0 \longrightarrow\left(S^{0}(M) \bar{\otimes} S\right)_{m} \longrightarrow\left(S^{0}(M) \bar{\otimes} F\right)_{m}
$$

is exact, and so is

$$
0 \longrightarrow M \otimes_{R} Z_{m}(S) \longrightarrow M \otimes_{R} Z_{m}(F)
$$

by the previous isomorphisms. Hence, $Z_{m}(S)$ is a pure submodule of $Z_{m}(F)$.

Now suppose $S$ is an exact subcomplex of $F$ such that $Z_{m}(S)$ is a pure submodule of $Z_{m}(F)$. Let $A$ be a complex in $\mathbf{C h}\left(\mathbf{M o d}_{R}\right)$. We want to show the sequence

$$
0 \longrightarrow A \bar{\otimes} S \longrightarrow A \bar{\otimes} F
$$

is exact. Since $Z_{n-k}(S)$ is a pure submodule of $Z_{n-k}(F)$, we have that the sequence

$$
0 \longrightarrow A_{k} \otimes_{R} Z_{n-k}(S) \longrightarrow A_{k} \otimes_{R} Z_{n-k}(F)
$$

is exact in ${ }_{R}$ Mod for all $k, n \in \mathbb{Z}$. Since $S$ and $F$ are exact complexes, we obtain the following commutative diagram where the top and the 
bottom rows are exact (recall Lemma 3.5):

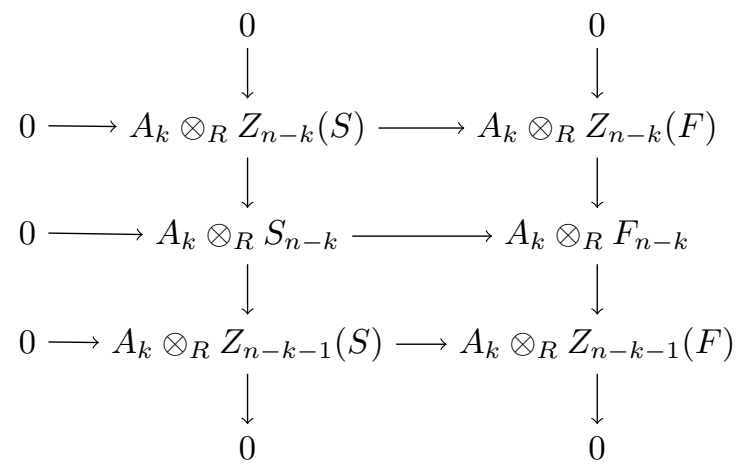

The columns of this diagram are also exact since the cycles $Z_{n-k}(S)$ and $Z_{n-k}(F)$ are flat modules. Since the class of short exact sequences is closed under extensions,

$$
0 \longrightarrow A_{k} \otimes_{R} S_{n-k} \longrightarrow A_{k} \otimes_{R} F_{n-k}
$$

is exact. It follows that

$$
0 \longrightarrow(A \otimes S)_{n} \longrightarrow(A \otimes F)_{n}
$$

is exact for every $n \in \mathbb{Z}$. Then

$$
0 \longrightarrow A \otimes S \longrightarrow A \otimes F
$$

is an exact sequence of complexes, and so each

$$
0 \longrightarrow B_{n}(A \otimes S) \longrightarrow B_{n}(A \otimes F)
$$

is exact in ${ }_{R}$ Mod. Since the class of short exact sequences is thick, we have that, for each $n \in \mathbb{Z}$, the sequence,

$$
0 \longrightarrow \frac{(A \otimes S)_{n}}{B_{n}(A \otimes S)} \longrightarrow \frac{(A \otimes F)_{n}}{B_{n}(A \otimes F)},
$$

is also exact in ${ }_{R}$ Mod. Hence,

$$
0 \longrightarrow A \bar{\otimes} S \longrightarrow A \bar{\otimes} F
$$

is exact in $\mathbf{C h}\left({ }_{R} \mathbf{M o d}\right)$. 
Recall from Section 4 that the extension groups $\operatorname{Ext}_{R}^{i}(M, N)$ can be computed by resolving $M$ by the class of $n$-projective modules. The arguments presented there carry over to the $n$-flat model structure, so $\operatorname{Ext}_{R}^{i}(M, N)$ can be obtained by resolving $M$ by $n$-flat modules, and $N$ by the class $\left(\mathcal{F}_{n}\right)^{\perp}$.

Consider the class $\mathcal{F}_{0}$ of flat modules. In [1], it is proven that the classes dw $\widetilde{\mathcal{F}_{0}}$ and ex $\widetilde{\mathcal{F}_{0}}$ are filtered by

$$
\left(\operatorname{dw} \widetilde{\mathcal{F}_{0}}\right) \leq \kappa \text { and }\left(\operatorname{ex} \widetilde{\mathcal{F}_{0}}\right) \leq \kappa,
$$

respectively. It follows that the pairs

$$
\left(\operatorname{dw} \widetilde{\mathcal{F}_{0}},\left(\operatorname{dw} \widetilde{\mathcal{F}_{0}}\right)^{\perp}\right) \text { and }\left(\operatorname{ex~} \widetilde{\mathcal{F}_{0}},\left(\operatorname{ex} \widetilde{\mathcal{F}_{0}}\right)^{\perp}\right)
$$

are complete. Since these two cotorsion pairs turn out to be compatible by Proposition 5.6, we have a unique Abelian model structure on $\mathbf{C h}\left({ }_{R} \mathbf{M o d}\right)$, which shall be referred to as the degreewise flat model structure, where the (trivial) cofibrations are monomorphisms with cokernels in $\mathrm{dw} \widetilde{\mathcal{F}_{0}}$ (in ex $\widetilde{\mathcal{F}_{0}}$ ), the (trivial) fibrations are the epimorphisms with kernels in $\left(\operatorname{ex} \widetilde{\mathcal{F}_{0}}\right)^{\perp}\left(\right.$ in $\left.\left(\operatorname{dw} \widetilde{\mathcal{F}_{0}}\right)^{\perp}\right)$, and the weak equivalences are quasi-isomorphisms. This model structure was discovered by Gillespie [10].

To extend this model structure to $n$-flat modules, we show that the induced cotorsion pairs $\left(\operatorname{dw} \widetilde{\mathcal{F}_{n}},\left(\operatorname{dw} \widetilde{\mathcal{F}_{n}}\right)^{\perp}\right)$ and $\left(\operatorname{ex} \widetilde{\mathcal{F}_{n}},\left(\operatorname{ex} \widetilde{\mathcal{F}_{n}}\right)^{\perp}\right)$ are complete, applying a modified version of the zigzag procedure, which also works to show the completeness of the pair $\left(\mathrm{dw} \widetilde{\mathcal{P}_{n}},\left(\mathrm{dw} \widetilde{\mathcal{P}_{n}}\right)^{\perp}\right)$, without assuming $R$ Noetherian as in Section 5 .

In what follows, we shall focus on proving that every chain complex in ex $\widetilde{\mathcal{F}_{n}}$ is filtered by the class $\left(\right.$ ex $\left.\widetilde{\mathcal{F}_{n}}\right) \leq \kappa$ of $\kappa$-small exact degreewise $n$-flat complexes. The proof of the following theorem is based on the arguments given in [1, Proposition 4.1] (where the authors proved the case $n=0)$.

Theorem 6.8. Let $X \in \operatorname{ex} \widetilde{\mathcal{F}_{n}}$ and $x \in X$, i.e., $x \in X_{m}$ for some $m \in \mathbb{Z}$. Then there exists an exact subcomplex $Y$ of $X$ with $x \in Y$ such that $Y_{m}$ is a $\kappa$-small and $n$-pure submodule of $X_{m}$, for every $m \in \mathbb{Z}$.

Proof. Assume, without loss of generality, that $x \in X_{0}$. Consider the submodule $\langle x\rangle \subseteq X_{0}$. Since $X_{0} \in \mathcal{F}_{n}$ and $\langle x\rangle$ is $\kappa$-small, we can 
embed $\langle x\rangle$ into a $\kappa$-small and $n$-pure submodule $Y_{0}^{1} \subseteq X_{0}$ (Lemma 6.4). We can construct a $\kappa$-small and exact subcomplex

$$
L^{1}:=\left(\cdots \longrightarrow L_{2}^{1} \longrightarrow L_{1}^{1} \longrightarrow Y_{0}^{1} \longrightarrow \partial_{0}\left(Y_{0}^{1}\right) \longrightarrow 0 \longrightarrow \cdots\right),
$$

since $X$ is exact. The fact that $\partial_{0}\left(Y_{0}^{1}\right)$ is $\kappa$-small implies that it is contained in a $\kappa$-small and $n$-pure submodule $Y_{-1}^{2} \subseteq X_{-1}$. As above, we can construct a $\kappa$-small and exact subcomplex of the form

$L^{2}:=\left(\cdots \longrightarrow L_{2}^{2} \longrightarrow L_{1}^{2} \longrightarrow L_{0}^{2} \longrightarrow Y_{-1}^{2} \longrightarrow \partial_{-1}\left(Y_{-1}^{2}\right) \longrightarrow 0 \longrightarrow \cdots\right)$.

Note that it is possible to construct $L^{2}$ containing $L^{1}$. Now embed $L_{0}^{2}$ into a $\kappa$-small and $n$-pure submodule $Y_{0}^{3} \subseteq X_{0}$. Again, construct a subcomplex

$$
\begin{aligned}
& L^{3}:=(\cdots \longrightarrow L_{2}^{3} \longrightarrow L_{1}^{3} \longrightarrow Y_{0}^{3} \longrightarrow Y_{-1}^{2}+\partial_{0}\left(Y_{0}^{3}\right)> \\
&\left.\longleftrightarrow \partial_{-1}\left(Y_{-1}^{2}\right) \longrightarrow 0 \longrightarrow \cdots\right)
\end{aligned}
$$

containing $L^{2}$, which is $\kappa$-small and exact. Now let $Y_{1}^{4}$ be a $\kappa$-small and $n$-pure submodule of $X_{1}$ containing $L_{1}^{3}$, and construct an exact and $\kappa$-small complex $L^{4}$ containing $L^{3}$ of the form

$$
\begin{aligned}
& L^{4}:=(\cdots \longrightarrow L_{2}^{4} \longrightarrow Y_{1}^{4} \rightarrow Y_{0}^{3}+\partial_{1}\left(Y_{1}^{4}\right) \rightarrow Y_{-1}^{2}+\partial_{0}\left(Y_{0}^{3}\right) \rightarrow \\
&\left.\longleftrightarrow \partial_{-1}\left(Y_{-1}^{2}\right) \longrightarrow 0 \longrightarrow \cdots\right)
\end{aligned}
$$

Embed $Y_{0}^{3}+\partial_{1}\left(Y_{1}^{4}\right)$ into a $\kappa$-small and $n$-pure submodule $Y_{0}^{5} \subseteq X_{0}$, and construct an exact and $\kappa$-small subcomplex

$$
\begin{aligned}
& L^{5}:=(\cdots \longrightarrow L_{2}^{5} \longrightarrow L_{1}^{5} \longrightarrow Y_{0}^{5} \longrightarrow Y_{-1}^{2}+\partial_{0}\left(Y_{0}^{5}\right)> \\
&\left.\longleftrightarrow \partial_{-1}\left(Y_{-1}^{2}\right) \longrightarrow 0 \longrightarrow \cdots\right)
\end{aligned}
$$

containing $L^{4}$. In a similar way, construct $\kappa$-small and exact complexes

$$
L^{6}:=\left(\cdots \longrightarrow L_{1}^{6} \longrightarrow L_{0}^{6} \longrightarrow Y_{-1}^{6} \longrightarrow \partial_{-1}\left(Y_{-1}^{6}\right) \longrightarrow 0 \longrightarrow \cdots\right)
$$

and

$$
L^{7}:=\left(\cdots \longrightarrow L_{1}^{7} \longrightarrow L_{0}^{7} \longrightarrow L_{-1}^{7} \longrightarrow Y_{-2}^{7} \longrightarrow \partial_{-2}\left(Y_{-2}^{7}\right) \longrightarrow 0 \longrightarrow \cdots\right)
$$


such that $Y_{-1}^{6}$ is a $\kappa$-small and $n$-pure submodule of $X_{-1}$ containing $Y_{-1}^{2}+\partial_{0}\left(Y_{0}^{5}\right)$, and $Y_{-2}^{7}$ is a $\kappa$-small and $n$-pure submodule of $X_{-2}$ containing $\partial_{-1}\left(Y_{-1}^{6}\right)$.

Keep repeating this procedure, and set

$$
Y:=\bigcup_{n \geq 1} L^{n}, \quad \text { where } \quad Y_{i}:=\bigcup_{n \geq 1}\left(L^{n}\right)_{i} .
$$

It is clear that $Y$ is an exact complex. We check that each $Y_{m}$ is a $\kappa$ small and $n$-pure submodule of $X_{m}$. It suffices to show the case $m=0$. We have

$$
\begin{aligned}
Y_{0} & =Y_{0}^{1} \cup L_{0}^{2} \cup Y_{0}^{3} \cup\left(Y_{0}^{3}+\partial_{1}\left(Y_{1}^{4}\right)\right) \cup Y_{0}^{5} \cup \cdots \\
& =Y_{0}^{1} \cup Y_{0}^{3} \cup Y_{0}^{5} \cup \cdots
\end{aligned}
$$

is $\kappa$-small. Consider a left flat resolution of length $n$,

$$
(1)=\left(0 \longrightarrow F_{n} \longrightarrow \cdots \longrightarrow F_{1} \longrightarrow F_{0} \longrightarrow X_{0} \rightarrow 0\right) .
$$

By Lemma 6.4, we can construct a subresolution

$$
0 \longrightarrow S_{n}^{1} \longrightarrow \cdots \longrightarrow S_{1}^{1} \longrightarrow S_{0}^{1} \longrightarrow Y_{0}^{1} \longrightarrow 0,
$$

where $\langle x\rangle \subseteq Y_{0}^{1}$, and each $S_{k}^{1}$ is a $\kappa$-small and pure submodule of $F_{k}$. As in the previous case, applying Lemma 6.4 infinitely many times, we can get an ascending chain of subresolutions

$$
\begin{aligned}
& 0 \longrightarrow S_{n}^{1} \longrightarrow \cdots \longrightarrow S_{1}^{1} \longrightarrow S_{0}^{1} \longrightarrow Y_{0}^{1} \longrightarrow 0 \\
& 0 \longrightarrow S_{n}^{3} \longrightarrow \cdots \longrightarrow S_{1}^{3} \longrightarrow S_{0}^{3} \longrightarrow Y_{0}^{3} \longrightarrow 0 \\
& 0 \longrightarrow S_{n}^{5} \longrightarrow \cdots \longrightarrow S_{1}^{5} \longrightarrow S_{0}^{5} \longrightarrow Y_{0}^{5} \longrightarrow 0, \ldots
\end{aligned}
$$

Taking the union of these subresolutions yields an exact sequence

$$
(2)=\left(0 \longrightarrow \bigcup_{j} S_{n}^{j} \longrightarrow \cdots \longrightarrow \bigcup_{j} S_{1}^{j} \longrightarrow \bigcup_{j} S_{0}^{j} \longrightarrow Y_{0} \longrightarrow 0\right),
$$

where each $\bigcup_{j} S_{k}^{j}$ is a $\kappa$-small and pure submodule of $F_{k}$ (so it is flat). have

If $Y$ is the subcomplex of $X$ given by the previous theorem, then we

$$
Y_{m} \in\left(\mathcal{F}_{n}\right)^{\leq \kappa} \quad \text { and } \quad X_{m} / Y_{m} \in \mathcal{F}_{n}
$$


(Take the quotient of (1) by (2).) It follows that

$$
Y \in\left(\operatorname{ex} \widetilde{\mathcal{F}_{n}}\right) \leq \kappa \quad \text { and } \quad X / Y \in \operatorname{ex} \widetilde{\mathcal{F}_{n}}
$$

Remark 6.9. The arguments given in the previous proof also show that every exact degreewise $n$-projective complex has a filtration by the class ex $\widetilde{\mathcal{P}_{n}(\kappa)}$. Proceed in the same way as in the previous proof, using Lemma 5.8, until $Y$ is constructed. Consider

$$
(1)=\left(0 \longrightarrow \bigoplus_{i \in I_{n}} P_{n}^{i} \longrightarrow \cdots \longrightarrow \bigoplus_{i \in I_{1}} P_{1}^{i} \longrightarrow \bigoplus_{i \in I_{0}} P_{0}^{i} \longrightarrow X_{0} \longrightarrow 0\right)
$$

a projective resolution of $X_{0}$ of length $n$, where each direct sum consists of countably generated projective modules. By Lemma 5.8, we can construct $Y_{0}^{1}$ containing $\langle x\rangle$ with a subresolution of the form

$$
(2)=\left(0 \longrightarrow \bigoplus_{i \in I_{n}^{1}} P_{n}^{i} \longrightarrow \cdots \longrightarrow \bigoplus_{i \in I_{1}^{1}} P_{1}^{i} \longrightarrow \bigoplus_{i \in I_{0}^{1}} P_{0}^{i} \longrightarrow Y_{0}^{1} \longrightarrow 0\right)
$$

where each $I_{k}^{1} \subset I_{k}$ is $\kappa$-small. Note that the quotient of (1) by (2) yields a projective resolution of $X_{0} / Y_{0}^{1}$ of length $n$, so $X_{0} / Y_{0}^{1} \in \mathcal{P}_{n}$. Using Lemma 5.8 again, we can construct a subresolution containing (2), say,

$$
(3)=\left(0 \longrightarrow \bigoplus_{i \in I_{n}^{3}} P_{n}^{i} \longrightarrow \cdots \longrightarrow \bigoplus_{i \in I_{1}^{3}} P_{1}^{i} \longrightarrow \bigoplus_{i \in I_{0}^{3}} P_{0}^{i} \longrightarrow Y_{0}^{3} \longrightarrow 0\right)
$$

such that $X_{0} / Y_{0}^{3} \in \mathcal{P}_{n}$. Keep applying Lemma 5.8 to get an ascending chain of subresolutions of (1):

$$
\begin{aligned}
& 0 \longrightarrow \bigoplus_{i \in I_{n}^{1}} P_{n}^{i} \longrightarrow \cdots \longrightarrow \bigoplus_{i \in I_{1}^{1}} P_{1}^{i} \longrightarrow \bigoplus_{i \in I_{0}^{1}} P_{0}^{i} \longrightarrow Y_{0}^{1} \longrightarrow 0 \\
& 0 \longrightarrow \bigoplus_{i \in I_{n}^{3}} P_{n}^{i} \longrightarrow \cdots \longrightarrow \bigoplus_{i \in I_{1}^{3}} P_{1}^{i} \longrightarrow \bigoplus_{i \in I_{0}^{3}} P_{0}^{i} \longrightarrow Y_{0}^{3} \longrightarrow 0 \\
& 0 \longrightarrow \bigoplus_{i \in I_{n}^{5}} P_{n}^{i} \longrightarrow \cdots \longrightarrow \bigoplus_{i \in I_{1}^{5}} P_{1}^{i} \longrightarrow \bigoplus_{i \in I_{0}^{5}} P_{0}^{i} \longrightarrow Y_{0}^{5} \longrightarrow 0, \ldots
\end{aligned}
$$


Now take the union of this ascending chain, and get the exact complex

$$
(4)=\left(0 \longrightarrow \bigoplus_{i \in \bigcup_{j} I_{n}^{j}} P_{n}^{i} \longrightarrow \cdots \longrightarrow \bigoplus_{i \in \bigcup_{j} I_{0}^{j}} P_{0}^{i} \longrightarrow Y_{0} \longrightarrow 0\right) .
$$

Since each $\bigcup_{j} I_{k}^{j}$ is a $\kappa$-small subset of $I_{k}$, we have that the previous sequence is a nice $\kappa$-small projective subresolution of (1). Note also that the quotient of (1) by (4) yields a projective resolution of $X_{0} / Y_{0}$ of length $n$. Then $Y_{0} \in \mathcal{P}_{n}(\kappa)$. In a similar way, we have that $Y_{m} \in \mathcal{P}_{n}(\kappa)$ and $X_{m} / Y_{m} \in \mathcal{P}_{n}$, for every $m \in \mathbb{Z}$.

By the previous result, we can use transfinite induction to show that every complex in ex $\widetilde{\mathcal{F}_{n}}$ is filtered by the class $\left(\right.$ ex $\left.\widetilde{\mathcal{F}_{n}}\right) \leq \kappa$. The same holds for the class $\mathcal{P}_{n}$. Using Proposition 2.5 and Eklof and Trlifaj's theorem, we have that

$$
\left(\operatorname{ex} \widetilde{\mathcal{P}_{n}},\left(\operatorname{ex} \widetilde{\mathcal{P}_{n}}\right)^{\perp}\right) \text { and }\left(\operatorname{ex} \widetilde{\mathcal{F}_{n}},\left(\operatorname{ex} \widetilde{\mathcal{F}_{n}}\right)^{\perp}\right)
$$

are complete cotorsion pairs. In a similar way, one can prove that the same holds for the pairs

$$
\left(\operatorname{dw} \widetilde{\mathcal{P}_{n}},\left(\operatorname{dw} \widetilde{\mathcal{P}_{n}}\right)^{\perp}\right) \text { and }\left(\operatorname{dw} \widetilde{\mathcal{F}_{n}},\left(\operatorname{dw} \widetilde{\mathcal{F}_{n}}\right)^{\perp}\right) .
$$

Furthermore,

$$
\left(\operatorname{dw} \widetilde{\mathcal{F}_{n}},\left(\operatorname{dw} \widetilde{\mathcal{F}_{n}}\right)^{\perp}\right) \text { and } \quad\left(\operatorname{ex} \widetilde{\mathcal{F}_{n}},\left(\operatorname{ex} \widetilde{\mathcal{F}_{n}}\right)^{\perp}\right)
$$

are compatible by Proposition 5.6. Therefore, Theorem 6.2 follows. Similarly, we have another proof of Theorem 5.1.

Remark 6.10. There are many examples of rings $R$ for which flat $R$ modules have finite projective dimension. In some of these cases, the $n$-projective model structures are related to the flat model structure, in the sense that every cofibrant object in the latter model structure is also cofibrant in the former one. This can be stated in a more formal way using the notion of Quillen functors.

Let

$$
F: \mathcal{C} \longrightarrow \mathcal{D} \text { and } \quad G: \mathcal{D} \longrightarrow \mathcal{C}
$$


be a pair of functors between model categories $\mathcal{C}$ and $\mathcal{D}$, and

$$
\varphi: \operatorname{Hom}_{\mathcal{D}}(F(-),-) \longrightarrow \operatorname{Hom}_{\mathcal{C}}(-, G(-))
$$

a natural isomorphism, i.e., $(F, G, \varphi)$ is an adjunction. The functor $F$ is a left Quillen functor if it preserves cofibrations and trivial cofibrations. The adjunction $(F, G, \varphi)$ is called a Quillen adjunction if $F$ is a left Quillen functor. Finally, a Quillen adjunction $(F, G, \varphi)$ is called a Quillen equivalence if, for all cofibrant $X$ in $\mathcal{C}$ and fibrant $Y$ in $\mathcal{D}$, a map

$$
f: F(X) \longrightarrow Y
$$

is a weak equivalence in $\mathcal{D}$ if and only if $\varphi(f): X \rightarrow G(Y)$ is a weak equivalence in $\mathcal{C}$.

(1) Let $R$ be a left perfect ring. Then a module is projective if and only if it is flat. In this case, the $n$-projective and $n$-flat model structures coincide, for every non-negative integer $n$.

(2) Let $R$ be a countable ring. By [17], every flat module has projective dimension 1. Notice that this implies that every (differential graded) flat complex is (differential graded) 1-projective. Set $\mathcal{C}$ and $\mathcal{D}$ as the category $\mathbf{C h}\left({ }_{R} \mathbf{M o d}\right)$ equipped with the flat and 1projective model structures, respectively. It follows that, if we set $F$ and $G$ as the identity on the objects and maps of $\mathbf{C h}\left({ }_{R} \mathbf{M o d}\right)$, then $(F, G)$ is a Quillen equivalence.

(3) The finitistic projective dimension of a ring $R$ is defined as the supremum

$\operatorname{FPD}(R):=\{\operatorname{pd}(M): M$ has finite projective dimension $\}$.

Let $R$ be a ring such that $\operatorname{FPD}(R)$ is finite, and let $n=\operatorname{FPD}(R)$. By [16], any $R$-module of finite flat dimension has finite projective dimension, in this case $\leq n$. Fix a non-negative integer $k \leq n$. Notice that every (differential graded) $k$-flat complex is (differential graded) $n$-projective. It follows that the $k$-flat and $n$-projective model category structures are Quillen equivalent.

We conclude this remark by noting that (1), (2), (3) and (4) are also valid for the corresponding degreewise homological model structures.

Gillespie showed in [10, subsection 5.2] that the degreewise flat model structure is not monoidal on $\left(\mathbf{C h}\left({ }_{R} \mathbf{M o d}\right), \otimes\right)$ in general. $\mathrm{He}$ 
considered the category of complexes over the ring $\mathbb{Z}_{4}$, where

$$
Y=\cdots \longrightarrow \mathbb{Z}_{4} \stackrel{\times 2}{\longrightarrow} \mathbb{Z}_{4} \stackrel{\times 2}{\longrightarrow} \mathbb{Z}_{4} \longrightarrow \cdots
$$

is an exact degreewise flat complex, but $Y \otimes Y \notin$ ex $\widetilde{\mathcal{F}_{0}}$, since it is not even exact. This counterexample also works to show that the degreewise $n$-flat model structure is not monoidal for every $n>0$.

One problem with the ring $\mathbb{Z}_{4}$ considered by Gillespie is that $\mathbb{Z}_{4}$ has infinite weak dimension. $\mathbb{Z}_{2}$ is a $\mathbb{Z}_{4}$-module with infinite flat dimension, since $\operatorname{Tor}_{k}^{\mathbb{Z}_{4}}\left(\mathbb{Z}_{2}, \mathbb{Z}_{2}\right) \cong \mathbb{Z}_{2} \neq 0$ for every $k \geq 0$ [20, Chapter 3 , Example 9]. This "inconvenience" can be solved if we impose an extra condition on $R$.

Proposition 6.11. Let $R$ be a commutative ring with weak dimension at most 1 . Then the degreewise projective and degreewise flat model structures are monoidal.

We know that $\left(\mathbf{C h}\left({ }_{R} \mathbf{M o d}\right), \otimes\right)$ is a closed symmetric monoidal category with unit $S^{0}(R)$. Flat objects in a monoidal category $(\mathcal{C}, \otimes)$ are defined as those $X \in \mathrm{Ob}(\mathcal{C})$ such that the functor $-\otimes X: \mathcal{C} \rightarrow \mathcal{C}$ is exact. Previously, we have considered flat objects in $\mathbf{C h}\left({ }_{R} \mathbf{M o d}\right)$ with respect to $\bar{\otimes}$. For the rest of this section, we shall say that a complex $F$ is flat with respect to $\otimes$ if the functor $-\otimes F$ is exact.

We only prove Proposition 6.11 for the flat case (since the projective case follows similarly). By [13, Theorem 4.2], it suffices to verify the following conditions:

(1) Every complex in $\mathrm{dw} \widetilde{\mathcal{F}_{0}}$ is flat with respect to $\otimes$.

(2) If $X, Y \in \mathrm{dw} \widetilde{\mathcal{F}_{0}}$, then so is $X \otimes Y$.

(3) If $X, Y \in \operatorname{dw} \widetilde{\mathcal{F}_{0}}$ and one of them is exact, then $X \otimes Y$ is exact.

(4) $S^{0}(R) \in \operatorname{dw} \widetilde{\mathcal{F}_{0}}$.

Note that condition (4) is immediate, and (2) is easy to verify. To show (1), we establish the following characterization.

Proposition 6.12. A chain complex $X \in \mathbf{C h}\left({ }_{R} \mathbf{M o d}\right)$ over a commutative ring $R$ is flat with respect to $\otimes$ if, and only if, it is degreewise flat. 
Proof. Let $Y$ be a chain complex such that $-\otimes Y$ is exact. Consider an exact sequence

$$
0 \longrightarrow A \longrightarrow B \longrightarrow C \longrightarrow 0 \text { in }{ }_{R} \operatorname{Mod} .
$$

Then we obtain an exact sequence

$0 \longrightarrow S^{0}(A) \otimes Y \longrightarrow S^{0}(B) \otimes Y \longrightarrow S^{0}(C) \otimes Y \longrightarrow 0 \quad$ in $\mathbf{C h}\left({ }_{R}\right.$ Mod $)$

So, for each $n \in \mathbb{Z}$, we have the short exact sequence

$$
0 \longrightarrow A \otimes_{R} Y_{n} \longrightarrow B \otimes_{R} Y_{n} \longrightarrow C \otimes_{R} Y_{n} \longrightarrow 0 .
$$

Now suppose $Y \in \mathrm{dw} \widetilde{\mathcal{F}_{0}}$. Consider a short exact sequence of chain complexes

$$
0 \longrightarrow A \stackrel{\alpha}{\longrightarrow} B \stackrel{\beta}{\longrightarrow} C \longrightarrow 0
$$

and apply $-\otimes Y$. We need to check that, for every $n \in \mathbb{Z}$, the sequence

$$
0 \longrightarrow(A \otimes Y)_{n} \stackrel{(\alpha \otimes Y)_{n}}{\longrightarrow}(B \otimes Y)_{n} \stackrel{(\beta \otimes Y)_{n}}{\longrightarrow}(C \otimes Y)_{n} \longrightarrow 0
$$

is exact. In other words, we shall see

$$
\begin{gathered}
(*)=\left(0 \longrightarrow \bigoplus_{k \in \mathbb{Z}} A_{k} \otimes_{R} Y_{n-k} \longrightarrow\right. \\
\left.\bigoplus_{k \in \mathbb{Z}} B_{k} \otimes_{R} Y_{n-k} \underset{\bigoplus_{k \in \mathbb{Z}} \alpha_{k} \otimes_{R} Y_{n-k} \otimes_{R} Y_{n-k}}{\longrightarrow} \bigoplus_{k \in \mathbb{Z}} C_{k} \otimes_{R} Y_{n-k} \longrightarrow 0\right)
\end{gathered}
$$

is exact. For every $k \in \mathbb{Z}$, the sequence

$$
0 \longrightarrow A_{k} \otimes_{R} Y_{n-k} \stackrel{\alpha_{k} \otimes_{R} Y_{n-k}}{\longrightarrow} B_{k} \otimes_{R} Y_{n-k} \stackrel{\beta_{k} \otimes Y_{n-k}}{\longrightarrow} C_{k} \otimes_{R} Y_{n-k} \longrightarrow 0
$$

is exact since $Y_{n-k}$ is flat. It follows $(*)$ is exact since the direct sum of exact sequences is exact. (Recall that homology commutes with direct sums.)

It is only left to verify (3). If $X$ and $Y$ are degreewise flat chain complexes over a commutative ring $R$ with weak dimension at most 1 , then by [20, Theorem 9.16] we have the following Künneth exact 
sequence for every $k \in \mathbb{Z}$ :

$$
\begin{gathered}
0 \longrightarrow \bigoplus_{i+j=k} H_{i}(X) \otimes H_{j}(Y) \longrightarrow H_{k}(X \otimes Y) \longrightarrow \\
\bigoplus_{i+j=k-1} \operatorname{Tor}_{1}^{R}\left(H_{i}(X), H_{j}(Y)\right) \longrightarrow 0
\end{gathered}
$$

If, in addition, $X$ or $Y$ is exact, then it follows that $X \otimes Y$ is also exact. Therefore, Proposition 6.11 follows.

Acknowledgments. The author would like to thank Professor André Joyal of the Department of Mathematics at UQÀM, for his support and suggestions, and his enlightening introduction to the theory of modules over ringoids. Special thanks to the referee for a careful reading of this paper and for providing constructive comments and help in improving its contents.

\section{REFERENCES}

1. S.T. Aldrich, E.E. Enochs, J.R. García Rozas and L. Oyonarte, Covers and envelopes in Grothendieck categories: Flat covers of complexes with applications, J. Alg. 243 (2001), 615-630.

2. S.T. Aldrich, E.E. Enochs, O.M.G. Jenda and L. Oyonarte, Envelopes and covers by modules of finite injective and projective dimensions, J. Alg. 242 (2001), $447-459$.

3. L. Bican, R. El Bashir and E.E. Enochs, All modules have flat covers, Bull. Lond. Math. Soc. 33 (2001), 385-390.

4. D. Bravo, E.E. Enochs, A.C. Iacob, O.M.G. Jenda and J. Rada, Cotorsion pairs in $C$ (R-Mod), Rocky Mountain J. Math. 42 (2012), 1787-1802.

5. W.G. Dwyer and J. Spalinski, Homotopy theories and model categories, in Handbook of algebraic topology, I.M. James, ed., Elsevier, New York, 1995.

6. P. Eklof and J. Trlifaj, How to make Ext vanish, Bull. Lond. Math. Soc. 33 (2001), 41-51.

7. E.E. Enochs and O.M.G. Jenda, Relative homological algebra, de Gruyter Expos. Math. 30, Berlin, 2000.

8. J.R. García Rozas, Covers and envelopes in the category of complexes of modules, Res. Not. Math. 407, Chapman \& Hall/CRC, Boca Raton, FL, 1999.

9. J. Gillespie, The flat model structure on $\mathrm{Ch}(R)$, Trans. Amer. Math. Soc. 356 (2004), 3369-3390.

10. Cotorsion pairs and degreewise homological model structures, Homol. Homot. Appl. 10 (2008), 283-304. 
11. R. Göbel and J. Trlifaj, Approximations and endomorphism algebras of modules, De Gruyter Expos. Math. 31, De Gruyter, Berlin, 2006.

12. A. Grothendieck, Sur quelques points d'algèbre homologique, Tohoku Math. J. 9 (1957), 119-221.

13. M. Hovey, Cotorsion pairs and model categories, in Contemporary mathematics, Amer. Math. Soc. 436 (2007), 277-296.

14. Cotorsion pairs, model category structures and representation theory, Math. Z. 241 (2002), 553-592.

15. , Model categories, Math. Surv. Mono. 63, American Mathematical Society, Providence, RI, 1999.

16. C.U. Jensen, On the vanishing of $\mathrm{lim}^{(i)}$, J. Alg. 15 (1970), 151-166.

17. C.U. Jensen, On homological dimensions of rings with countably generated ideals, Math. Scand. 18 (1966), 97-105.

18. I. Kaplansky, Projective modules, Ann. Math. 68 (1958), 372-377.

19. S. Mac Lane, Categories for the working mathematician, Grad. Texts Math. 5, Springer Verlag, New York, 1978.

20. M.S. Osborne, Basic homological algebra, Grad. Texts Math. 196, Springer Verlag, New York, 2000.

21. L. Salce, Cotorsion theories for Abelian groups, Symp. Math. 23 (1979), $11-32$.

Instituto de Matemáticas, Universidad NaCional Autónoma de México, Área de la Investigación Científica, Circuito Exterior, Ciudad UniverSitaria, CoyoacÁn, 04510, MeXico City, MeXico

Email address: maperez@im.unam.mx, marco.perez@cirget.ca 